Portland State University

PDXScholar

$1-1-2020$

\title{
Analytical and field verification of a 3D hydrodynamic and water quality numerical scheme based on the 2D formulation in CE-QUAL-W2
}

Hussein Ali Mahdi Al-Zubaidi

Portland State University

Scott A. Wells

Portland State University, wellss@pdx.edu

Follow this and additional works at: https://pdxscholar.library.pdx.edu/cengin_fac

Part of the Hydraulic Engineering Commons

Let us know how access to this document benefits you.

\section{Citation Details}

Hussein A. M. Al-Zubaidi \& Scott A. Wells (2020) Analytical and field verification of a 3D hydrodynamic and water quality numerical scheme based on the 2D formulation in CE-QUAL-W2, Journal of Hydraulic Research, 58:1, 152-171, DOI: 10.1080/00221686.2018.1499051

This Post-Print is brought to you for free and open access. It has been accepted for inclusion in Civil and Environmental Engineering Faculty Publications and Presentations by an authorized administrator of PDXScholar. Please contact us if we can make this document more accessible: pdxscholar@pdx.edu. 


\section{Analytical and Field Verification of a 3D Hydrodynamic and Water Quality Numerical Scheme Based on the 2D Formulation in CE-QUAL-W2}

HUSSEIN A. M. AL-ZUBAIDI, PHD student, Department of Civil and Environmental Engineering, Portland State University, Portland, Oregon, USA; and Lecturer, Department of Environmental Engineering, University of Babylon, Babylon, Iraq

Email: alzubaidih10@gmail.com (author for correspondence)

SCOTT A. WELLS, Professor, Department of Civil and Environmental Engineering, Portland State University, Portland, Oregon, USA; and Collaborative Center for Geo-hazards and Eco-Environment in Three Gorges Area, Three Gorges University, Yichang, China

Email:wellss@pdx.edu

Running Head (3D hydrodynamic and water quality numerical scheme) 


\section{Analytical and Field Verification of a 3D Hydrodynamic and Water Quality Numerical Scheme Based on the 2D Formulation in CE-QUAL-W2 \\ ABSTRACT}

A new 3D hydrodynamic model was developed to simulate water quality transport in surface waterbodies. The governing equations are the continuity equation, free surface equation, momentum equation, and transport equation. The 2D numerical scheme of CE-QUAL-W2 was expanded in three dimensions and modified to solve for the free surface elevation. A time splitting technique was employed to solve the momentum and transport equation. The numerical formulation of the 3D scheme used a novel solution, which resulted in a tri-diagonal matrix form for solving the free surface equation rather than a more computationally intensive penta-diagonal matrix solution. In addition, the hydrodynamic and water quality equations were solved at the same time step in order to allow feedback between water quality and hydrodynamics. The verification of the model hydrodynamics and temperature was performed by comparing the model predictions to known analytical solutions and field data from Lake Chaplain, Washington, USA. There was good agreement of the solution of the hydrodynamic equations to analytical solutions and field data.

Keywords: CE-QUAL-W2; free surface flow; hydrodynamic model; lakes and reservoirs; numerical model verification; three-dimensional models; water quality 


\section{Introduction}

Many 3D hydrodynamic and water quality models have been developed since the 1960s. Different numerical solution techniques have been used to solve the governing equations depending on the model complexity. Each approach has its advantages and disadvantages depending on the numerical scheme implemented.

Most of the early group of 3D models used the mode splitting finite difference techniques to solve the governing equations. Blumberg and Mellor (1987) developed a three-dimensional numerical model for estuarine and coastal ocean circulation. The model was based on the vertical $\sigma$-coordinate and solved the continuity equation, free surface equation, Reynolds momentum equations, and conservation equations for temperature and salinity. The mode splitting technique separated the governing equations into an external and internal mode. In the external mode, shallow water wave equations were obtained by integrating the governing equations vertically and then solving explicitly in a short time step to satisfy the gravity wave Courant-Friedrichs-Levy or CFL limitation. The free surface elevation resulting from the external mode was then used to solve the internal mode, the original governing equations, in a long time step independently from the external mode by treating just the vertical diffusion terms implicitly. This technique helped the stability of the internal mode to not be affected by the gravity wave stability, leading to a much longer time step than the internal mode. Finally, the internal mode produced a tri-diagonal system of linear equations which were then solved by a Gaussian elimination method. Different experiments were done for testing the model performance by making comparisons with field data. This model has been developed by several authors since 1987 when the original model later became the POM, the Princeton Ocean Model (Mellor, 2002). In the late 1990s and the 2000s, many threedimensional models have been derived from POM such as ECOM (A F Blumberg \& Mellor, 1987; A. F. Blumberg \& Mellor, 1980), NCOM (Barron, Kara, Martin, Rhodes, \& Smedstad, 2006; Martin et al., 2009), and FVCOM (Chen et al., 2011; Chen, Liu, \& Beardsley, 2003).

Hamrick (1992) developed the Environmental Fluid Dynamic Code, EFDC, which is a threedimensional model similar to Blumberg and Mellor (1987) in its physics and many aspects of the computational scheme. The main differences between the two models are the internal and external mode solution of EFDC model is computed at the same model time step. The EFDC model also implements a number of alternate advection transport schemes, such as the central time central space scheme and the forward time upwind space scheme. A further development of EFDC model led to the model EFDCHydro, a special version developed for U.S. EPA Region 4 (Tetra Tech, 2002). The model verification was performed by comparing the numerical solution with field data and calculating the error.

Another group of 3D models is based on the semi-implicit finite difference technique. Casulli and Cheng (1992) developed a three-dimensional numerical model for shallow water flow (TRIM-3D). The 
governing equations were derived from the Navier-Stokes equations based on turbulent averaging and assuming a constant density and hydrostatic pressure. The non-conservative forms of vertically averaged horizontal and vertical momentum equations, free surface equation, and continuity equation were solved without coordinate transformations. The numerical solution was based on fixed staggered grids with a semi-implicit finite difference method and an Eulerian-Lagrangian method for the convective terms. The vertical diffusion terms were solved implicitly, and the horizontal diffusion terms were solved explicitly. The model stability condition depended on the horizontal viscosity. The numerical solution yielded two types of linear systems: a tri-diagonal matrix from the numerical solution of the horizontal velocities and a penta-diagonal matrix from the numerical solution of the free surface equation. The model was verified and calibrated by implementing it on two different case studies. Also, Casulli and Walters (2000) developed an unstructured grid version of this model, UnTRIM.

Different models have been developed based on the model of Casulli and Cheng (1992). The most well-known is ELCOM by Hodges and Dallimore (2006) to simulate hydrodynamics and water quality in surface waters. The fundamental numerical scheme was based on the model of Casulli and Cheng (1992) with some adjustments relevant to accuracy, scalar conservation, numerical diffusion, and a new option for calculating vertical turbulent fluxes by application of a mixed-layer turbulence closure scheme. Using the mixed-layer scheme eliminated solving a tri-diagonal matrix for each water column, whereas the advection terms in hydrodynamic equations were treated similar to TRIM model by using a conservative third-order scalar transport method (ULTIMATE QUICKEST) proposed by Leonard (1991) was used. Ahsan and Blumberg (1999) developed a three-dimensional numerical model for simulating the dynamic and thermal distribution in Onondaga Lake, New York. This model, called ECOMsiz, was another version of the Blumberg and Mellor (1987) model, called ECOM. ECOMsiz employed the z-coordinate system and used the semi-implicit finite difference method of Casulli and Cheng (1992). Thus, the stability condition depended only on the horizontal viscosity and the solution scheme of the convective terms using an explicit discretization. Two years of data, 1985 and 1989, were used to validate ECOMsiz in Ahsan and Blumberg (1999).

Furthermore, Edinger (2001) developed the GLLVHT model, the generalized longitudinal lateral vertical hydrodynamic and transport model. This model was developed by coupling the momentum equations with the free surface equation to solve for the water level and velocity field. The solution algorithm was similar to TRIM-3D model, but the z-coordinate system was employed using constant depth increments in addition to using the Von Karman model for determining the vertical diffusion coefficient of momentum.

Another way to eliminate the stability related to gravity waves and provide a long time step for a large scale current system economically was illustrated in Bryan (1969) where gravity waves were filtered out 
of the solution by using a "rigid-lid" approximation. For oceanic circulation, this method may still be adequate, but for lakes and reservoirs under variable wind in space and time, this approach did not reproduce realistic results (Smith, 2006).

Wang and Falconer (1998) simulated the flow and disinfection processes in disinfection contact tanks by developing a three-dimensional model. Reynolds-averaged equations of continuity and momentums were integrated vertically and then free and bottom boundary conditions and different turbulent closure models were applied. The numerical solution was based on a time marching method, an alternatingdirections-implicit scheme. In an attempt to remove the numerical diffusion resulting from using the firstorder accurate upwind scheme, higher-order upwind schemes (QUICK and a third order-upwind scheme) were used in addition to the first-order accurate scheme. This model was validated and investigated by comparing model results with physical model tank results. The mesh consisted of a $49 \times 24$ grid with a spatial resolution of $0.043 \mathrm{~m} \times 0.042 \mathrm{~m}$. Various combinations of turbulent closure models and upwind schemes were investigated. The results showed that each combination has advantages and disadvantages.

The need to develop a new 3D model arises based on the following issues related to water quality models used in practice for reservoir systems: (1) Most 3D models do not couple water quality and hydrodynamics, hence there is no feedback between hydrodynamics and water quality processes affecting density such as algae growth and suspended solids through effects on light penetration. (2) Many schemes use the traditional penta-diagonal matrix form for the solution of the free surface equation. An efficient solution scheme for the free surface elevation can be used resulting in a tri-diagonal matrix form allowing use of the Thomas algorithm. (3) Most 3D models used for reservoirs do not include selective withdrawal theory (Imberger \& Fischer, 1970). This is an important algorithm to use for dam/reservoir withdrawals since it informs the model as to the vertical layers of the withdrawal without needing to solve the nearfield dynamics with the vertical momentum equation.

Hence, a new 3D model addressing these issues was developed. Since the 2D CE-QUAL-W2 model (Cole \& Wells, 2015) has been well-tested and verified on hundreds of lakes and reservoirs simulating thermal structure and water quality, the CE-QUAL-W2 algorithms used for turbulence transport, heat transfer, and water quality were also added to this new 3D model. Therefore, the main purpose of this article is to validate the predictions of a new 3D hydrodynamic and water quality numerical scheme by comparing model predictions to a series of analytical test cases and to field data from a stratified lake. 


\section{Model description}

\subsection{Hydrodynamic governing equations}

The three-dimensional hydrodynamic governing equations are derived from the Navier-Stokes equations. After time averaging the Navier-Stokes equations, we obtain the Reynolds-averaged equations (CushmanRoisin \& Beckers, 2007), which are as follows (Eqs 1, 2, 3, 4, and 5):

$$
\begin{gathered}
\frac{\partial \rho}{\partial t}+\frac{\partial \rho \bar{u}}{\partial x}+\frac{\partial \rho \bar{v}}{\partial y}+\frac{\partial \rho \bar{w}}{\partial z}=0 \\
\frac{\partial \rho \bar{u}}{\partial t}+\rho f_{*} \bar{w}-\rho f \bar{v}=-\frac{\partial \bar{p}}{\partial x}+\frac{\partial\left(\tau^{x x}+\tau_{x x}\right)}{\partial x}+\frac{\partial\left(\tau^{x y}+\tau_{x y}\right)}{\partial y}+\frac{\partial\left(\tau^{x z}+\tau_{x z}\right)}{\partial z}=0 \\
\frac{\partial \rho \bar{v}}{\partial t}+\rho f \bar{u}=-\frac{\partial \bar{p}}{\partial y}+\frac{\partial\left(\tau^{y x}+\tau_{y x}\right)}{\partial x}+\frac{\partial\left(\tau^{y y}+\tau_{y y}\right)}{\partial y}+\frac{\partial\left(\tau^{y z}+\tau_{y z}\right)}{\partial z}=0 \\
\frac{\partial \rho \bar{w}}{\partial t}+\rho f_{*} \bar{u}=-\frac{\partial \bar{p}}{\partial z}-\rho g \frac{\partial\left(\tau^{z x}+\tau_{z x}\right)}{\partial x}+\frac{\partial\left(\tau^{z y}+\tau_{z y}\right)}{\partial y}+\frac{\partial\left(\tau^{z z}+\tau_{z z}\right)}{\partial z}=0 \\
\frac{d}{d t}=\frac{\partial}{\partial t}+\bar{u} \frac{\partial}{\partial x}+\bar{v} \frac{\partial}{\partial y}+\bar{w} \frac{\partial}{\partial z}
\end{gathered}
$$

where $\bar{u}, \bar{v}$, and $\bar{w}$ are the average velocity in the $x, y$, and z-direction, respectively, $\rho$ is the density, $f=2 \Omega \sin (\varphi)$, called Coriolis parameter (positive above the equator, zero at the equator, and negative under the equator), $f_{*}=2 \Omega \cos (\varphi)$, called reciprocal Coriolis parameter (positive in both hemispheres and approaches to zero at the poles), $\Omega$ is the rotation rate of the earth, $\varphi$ is the latitude, $p$ is the pressure, $g$ is the gravitational acceleration, $\tau^{x x}, \tau^{x y}, \tau^{x z}, \tau^{y x}, \tau^{y y}, \tau^{y z}, \tau^{z x}, \tau^{z y}$, and $\tau^{z z}$ are the viscous shear stresses, and $\tau_{x x}, \tau_{x y}, \tau_{x z}, \tau_{y x}, \tau_{y y}, \tau_{y z}, \tau_{z x}, \tau_{z y}$, and $\tau_{z z}$ are turbulent shear stresses or Reynolds stresses.

The coordinates system was assumed as shown in Fig. 1 . The $x$-axis is at the free water surface, positive to the right in the flow direction, the $y$-axis is also at the free water surface, and the $z$-axis is the vertical axis, positive downward. The tangent of the angle $\alpha$ is the slope of the waterbody in case of rivers modeling. In addition, the main stream of the waterbody ( $x$-direction) makes an angle $\theta_{2}$ with the north direction.

\subsection{Hydrodynamic governing equations simplification}

\section{Assumptions}

We will make the following assumptions: The fluid is incompressible, centripetal acceleration is used as an adjustment to gravity, Boussinesq approximation of turbulence is valid, the reciprocal Coriolis 
parameter $(f *)$ is neglected, and the viscous shear stresses are neglected except at the boundaries where the turbulent shear stresses go to zero. Applying these assumptions to the continuity and momentum equations and rearranging terms, we obtain the turbulent time averaged equations (Eqs 6, 7, 8, and 9):

$$
\begin{gathered}
\frac{\partial \bar{u}}{\partial x}+\frac{\partial \bar{v}}{\partial y}+\frac{\partial \bar{w}}{\partial z}=0 \\
\frac{\partial \bar{u}}{\partial t}+\bar{u} \frac{\partial \bar{u}}{\partial x}+\bar{v} \frac{\partial \bar{u}}{\partial y}+\bar{w} \frac{\partial \bar{u}}{\partial z}=-\frac{1}{\rho_{\circ}} \frac{\partial \bar{p}}{\partial x}-f \bar{v}+\frac{1}{\rho_{\circ}} \frac{\partial\left(\tau_{x x}\right)}{\partial x}+\frac{1}{\rho_{\circ}} \frac{\partial\left(\tau_{x y}\right)}{\partial y}+\frac{1}{\rho_{\circ}} \frac{\partial\left(\tau_{x z}\right)}{\partial z} \\
\frac{\partial \bar{v}}{\partial t}+\bar{u} \frac{\partial \bar{v}}{\partial x}+\bar{v} \frac{\partial \bar{v}}{\partial y}+\bar{w} \frac{\partial \bar{v}}{\partial z}=-\frac{1}{\rho_{\circ}} \frac{\partial \bar{p}}{\partial y}+f \bar{u}+\frac{1}{\rho_{\circ}} \frac{\partial\left(\tau_{y x}\right)}{\partial x}+\frac{1}{\rho_{\circ}} \frac{\partial\left(\tau_{y y}\right)}{\partial y}+\frac{1}{\rho_{\circ}} \frac{\partial\left(\tau_{y z}\right)}{\partial z} \\
\frac{\partial \bar{w}}{\partial t}+\bar{u} \frac{\partial \bar{w}}{\partial x}+\bar{v} \frac{\partial \bar{w}}{\partial y}+\bar{w} \frac{\partial \bar{w}}{\partial z}=-\frac{1}{\rho_{\circ}} \frac{\partial \bar{p}}{\partial z}-\frac{\rho g}{\rho_{\circ}}+\frac{1}{\rho_{\circ}} \frac{\partial\left(\tau_{z x}\right)}{\partial x}+\frac{1}{\rho_{\circ}} \frac{\partial\left(\tau_{z y}\right)}{\partial y}+\frac{1}{\rho_{\circ}} \frac{\partial\left(\tau_{z z}\right)}{\partial z}
\end{gathered}
$$

\section{Reynolds stresses}

Reynolds stresses can be written in the simplest approach in $i$, and $j$ notation form (Eq. 10):

$$
\frac{\tau_{i j}}{\rho_{\circ}}=-u_{i}^{\prime} u_{j}^{\prime}=v_{t j} \frac{\partial u_{i}}{\partial x_{j}}
$$

where $u_{i}$ is the velocity component in the $i$-direction, $u^{\prime}$ is the fluctuation of the velocity component, $j=1$, 2 , and 3 are the coordinate system $x, y$, and $z$-direction respectively, and $v_{t j}$ is the turbulent kinematic viscosity in the $j$-direction.

If we substitute the Reynolds stresses in the momentum equations and use turbulent kinematic viscosities in only the horizontal ( $x$ and $y$ ) and vertical ( $z$ ) directions, the momentum equations in the three directions can be written as follows (Eqs 11, 12, and 13):

$$
\begin{gathered}
\frac{\partial \bar{u}}{\partial t}+\bar{u} \frac{\partial \bar{u}}{\partial x}+\bar{v} \frac{\partial \bar{u}}{\partial y}+\bar{w} \frac{\partial \bar{u}}{\partial z}=-\frac{1}{\rho_{o}} \frac{\partial \bar{p}}{\partial x}+f \bar{v}+\frac{\partial}{\partial x}\left[v_{h} \frac{\partial \bar{u}}{\partial x}\right]+\frac{\partial}{\partial y}\left[v_{h} \frac{\partial \bar{u}}{\partial y}\right]+\frac{\partial}{\partial z}\left[v_{v} \frac{\partial \bar{u}}{\partial z}\right] \\
\frac{\partial \bar{v}}{\partial t}+\bar{u} \frac{\partial \bar{v}}{\partial x}+\bar{v} \frac{\partial \bar{v}}{\partial y}+\bar{w} \frac{\partial \bar{v}}{\partial z}=-\frac{1}{\rho_{o}} \frac{\partial \bar{p}}{\partial y}-f \bar{u}+\frac{\partial}{\partial x}\left[v_{h} \frac{\partial \bar{v}}{\partial x}\right]+\frac{\partial}{\partial y}\left[v_{h} \frac{\partial \bar{v}}{\partial y}\right]+\frac{\partial}{\partial z}\left[v_{v} \frac{\partial \bar{v}}{\partial z}\right] \\
\frac{\partial \bar{w}}{\partial t}+\bar{u} \frac{\partial \bar{w}}{\partial x}+\bar{v} \frac{\partial \bar{w}}{\partial y}+\bar{w} \frac{\partial \bar{w}}{\partial z}=-\frac{1}{\rho_{o}} \frac{\partial \bar{p}}{\partial z}-\frac{\rho g}{\rho_{o}}+\frac{\partial}{\partial x}\left[v_{h} \frac{\partial \bar{w}}{\partial x}\right]+\frac{\partial}{\partial y}\left[v_{h} \frac{\partial \bar{w}}{\partial y}\right]+\frac{\partial}{\partial z}\left[v_{v} \frac{\partial \bar{w}}{\partial z}\right]
\end{gathered}
$$


where $v_{h}$ is the horizontal turbulent kinematic viscosity, and $v_{v}$ is the vertical turbulent kinematic viscosity.

\section{Gravitational acceleration components}

Assuming there is no change in the bottom elevation, $h$, with $y$-axis $(\partial h / \partial y=0)$, the gravitational acceleration, $g$, can be resolved into two components. These components increase the momentum in the $x$ and $z$-direction. Thus, one of these components is in the $x$-axis direction, and the other is in the $z$-axis direction (Eq. 14).

$$
g_{x}=-g \frac{\partial h}{\partial x}=g \sin \alpha \quad g_{z}=-g \frac{\partial h}{\partial z}=g \cos \alpha
$$

Adding the two components of the gravitational acceleration to the momentum equations, the momentums equations become:

$$
\begin{aligned}
& \frac{\partial \bar{u}}{\partial t}+\bar{u} \frac{\partial \bar{u}}{\partial x}+\bar{v} \frac{\partial \bar{u}}{\partial y}+\bar{w} \frac{\partial \bar{u}}{\partial z}=-\frac{1}{\rho_{\circ}} \frac{\partial \bar{p}}{\partial x}+f \bar{v}+g \sin \alpha+\frac{\partial}{\partial x}\left[v_{h} \frac{\partial \bar{u}}{\partial x}\right]+\frac{\partial}{\partial y}\left[v_{h} \frac{\partial \bar{u}}{\partial y}\right]+\frac{\partial}{\partial z}\left[v_{v} \frac{\partial \bar{u}}{\partial z}\right] \\
& \frac{\partial \bar{v}}{\partial t}+\bar{u} \frac{\partial \bar{v}}{\partial x}+\bar{v} \frac{\partial \bar{v}}{\partial y}+\bar{w} \frac{\partial \bar{v}}{\partial z}=-\frac{1}{\rho_{\circ}} \frac{\partial \bar{p}}{\partial y}-f \bar{u}+\frac{\partial}{\partial x}\left[v_{h} \frac{\partial \bar{v}}{\partial x}\right]+\frac{\partial}{\partial y}\left[v_{h} \frac{\partial \bar{v}}{\partial y}\right]+\frac{\partial}{\partial z}\left[v_{v} \frac{\partial \bar{v}}{\partial z}\right] \\
& \frac{\partial \bar{w}}{\partial t}+\bar{u} \frac{\partial \bar{w}}{\partial x}+\bar{v} \frac{\partial \bar{w}}{\partial y}+\bar{w} \frac{\partial \bar{w}}{\partial z}=-\frac{1}{\rho_{\circ}} \frac{\partial \bar{p}}{\partial z}+\frac{\rho g}{\rho_{\circ}} \cos \alpha+\frac{\partial}{\partial x}\left[v_{h} \frac{\partial \bar{w}}{\partial x}\right]+\frac{\partial}{\partial y}\left[v_{h} \frac{\partial \bar{w}}{\partial y}\right]+\frac{\partial}{\partial z}\left[v_{v} \frac{\partial \bar{w}}{\partial z}\right]
\end{aligned}
$$

\section{The hydrostatic assumption}

When the horizontal accelerations are larger than the vertical accelerations, a scaling analysis of the $z$ momentum equation shows that all terms can be cancelled out except the first and second term in the right side of (Eq. 17) (Cushman-Roisin \& Beckers, 2007). Thus, the z-momentum equation can be written in a new form (Eq. 18):

$$
0=-\frac{1}{\rho_{\circ}} \frac{\partial \bar{p}}{\partial z}+\frac{\rho g}{\rho_{\circ}} \cos \alpha
$$

Solving this first-order differential equation (Eq. 18) leads to the following solution (Eq. 19):

$$
\bar{p}=\overline{p_{a}}+g \cos \alpha \int_{\eta}^{z} \rho d z
$$

where $\eta$ is the free surface elevation, and $\overline{p_{a}}$ is the atmospheric pressure at the free surface elevation. By taking the derivative of the pressure (Eq. 19) with respect to $x$ and $y$ and applying Leibnitz's rule, we get a new expression for the pressure gradient in $x$ and $y$-direction (Eq. 20): 


$$
\begin{aligned}
& \frac{\partial \bar{p}}{\partial x}=\frac{\partial \overline{p_{a}}}{\partial x}+g \cos \alpha \int_{\eta}^{z} \frac{\partial \rho}{\partial x} d z-\rho g \cos \alpha \frac{\partial \eta}{\partial x} \\
& \frac{\partial \bar{p}}{\partial y}=\frac{\partial \overline{p_{a}}}{\partial y}+g \cos \alpha \int_{\eta}^{z} \frac{\partial \rho}{\partial y} d z-\rho g \cos \alpha \frac{\partial \eta}{\partial y}
\end{aligned}
$$

Assuming there is no change in the atmospheric pressure $\left(\partial \bar{p}_{a} / \partial x\right)=\left(\partial \overline{p_{a}} / \partial y\right)=0$ and then by substituting the pressure terms (Eq. 20) into the $x$ and $y$-momentum equation (Eqs. 15 and 16), respectively, the final governing momentum equations become:

$$
\begin{aligned}
& \frac{\partial \bar{u}}{\partial t}+\bar{u} \frac{\partial \bar{u}}{\partial x}+\bar{v} \frac{\partial \bar{u}}{\partial y}+\bar{w} \frac{\partial \bar{u}}{\partial z}= g \cos \alpha \frac{\partial \eta}{\partial x}-\frac{g \cos \alpha}{\rho_{\circ}} \int_{\eta}^{z} \frac{\partial \rho}{\partial x} d z+f \bar{v}+g \sin \alpha \\
&+\frac{\partial}{\partial x}\left[v_{h} \frac{\partial \bar{u}}{\partial x}\right]+\frac{\partial}{\partial y}\left[v_{h} \frac{\partial \bar{u}}{\partial y}\right]+\frac{\partial}{\partial z}\left[v_{v} \frac{\partial \bar{u}}{\partial z}\right] \\
& \frac{\partial \bar{v}}{\partial t}+\bar{u} \frac{\partial \bar{v}}{\partial x}+\bar{v} \frac{\partial \bar{v}}{\partial y}+\bar{w} \frac{\partial \bar{v}}{\partial z}= g \cos \alpha \frac{\partial \eta}{\partial y}-\frac{g \cos \alpha}{\rho_{\circ}} \int_{\eta}^{z} \frac{\partial \rho}{\partial y} d z-f \bar{u} \\
&+ \frac{\partial}{\partial x}\left[v_{h} \frac{\partial \bar{v}}{\partial x}\right]+\frac{\partial}{\partial y}\left[v_{h} \frac{\partial \bar{v}}{\partial y}\right]+\frac{\partial}{\partial z}\left[v_{v} \frac{\partial \bar{v}}{\partial z}\right] \\
& \frac{\partial \bar{p}}{\partial z}=\rho g \cos \alpha
\end{aligned}
$$

\section{Free surface equation}

The free surface equation (Eq. 24) can be derived by integrating the continuity equation over the total depth (see Fig. 2 for free surface integration limits) and applying kinematic boundary conditions derived from a mass balance at the surface and bottom layer of the waterbody.

$$
\frac{\partial \eta}{\partial t}=\frac{\partial}{\partial x} \int_{\eta}^{h} \bar{u} d z+\frac{\partial}{\partial y} \int_{\eta}^{h} \bar{v} d z
$$

\section{Boundary shear stresses}

Surface shear stresses, or wind shear stresses, are connected to the surface boundary conditions $(z=\eta)$.

These stresses are related to the wind velocity distribution above the waterbody and can be described as follows (Eq. 25) (Csanady, 2013; Wu, 1969):

$$
\tau_{s}=\rho_{a} C_{D} U|U| \quad U=W_{h}-U_{s}
$$


where $\tau_{s}$ is the surface shear stress, $\rho_{a}$ is the air density, $C_{D}$ is the drag coefficient, $W_{h}$ is the wind velocity at height $h$ (usually is taken at $10 \mathrm{~m}$ height), and $U_{s}$ is the surface shear velocity.

Because $W_{h} \gg U_{s}, U$ is assumed equal to $W_{h}$, and the surface shear stresses can be written as follows (Eq. 26) after analysis the wind velocity into two components:

$$
\left(\tau_{s x}, \tau_{s y}\right)=\rho_{a} C_{D}\left(W_{x}, W_{y}\right) \sqrt{W_{x}^{2}+W_{y}^{2}}
$$

where $\tau_{s x}$ and $\tau_{s y}$ are the surface shear stresses in the $x$ and $y$-direction, respectively, $W_{x}$ and $W_{y}$ are the wind velocities in the $x$ and $y$-direction, respectively, measured at $10 \mathrm{~m}$ height above the free surface elevation. If the measured wind height is at a different elevation than $10 \mathrm{~m}$, we can calculate the wind velocity at $10 \mathrm{~m}$ from the following equation (Eq. 27) (Ryan \& Harleman, 1973):

$$
\frac{W_{z}}{W_{z 1}}=\frac{\ln \left(\frac{Z}{Z_{\circ}}\right)}{\ln \left(\frac{z 1}{Z_{\circ}}\right)}
$$

where $W_{z}$ is the wind velocity at elevation $z, W_{z 1}$ is the wind velocity at elevation $z 1$, and $z \circ$ is the wind roughness height ( 0.003 for wind velocity less than $2.2 \mathrm{msec}^{-1}$ and 0.015 for wind velocity greater than $2.2 \mathrm{msec}^{-1}$ ). In addition, the wind shear stresses can be written depending on the angle that wind makes with the northern direction (Eq. 28):

$$
\left(\tau_{s x}, \tau_{s y}\right)=\rho_{a} C_{D} W_{h}^{2}(\cos \theta, \sin \theta) \quad \theta=\theta_{1}-\theta_{2}
$$

where $\theta_{1}$ is the angle that wind makes with the northern direction in radians (measured clockwise from the north) and $\theta_{2}$ is the angle that the $x$-direction makes with the northern direction in radians (measured clockwise from the north).

Bottom shear stresses at $z=h$, or wall shear stresses, are calculated depending on the horizontal velocities that are just above the bottom from the following equation (Eq. 29) (Dronkers, 1964):

$$
\tau_{b}=\frac{\rho_{w} g}{C_{z}^{2}} U|U|
$$

where $\tau_{b}$ is the bottom shear stress, $\rho_{w}$ is the water density, and $C_{z}$ is the Chezy friction coefficient. Thus, the bottom shear stresses ( $\tau_{b x}$ and $\tau_{b y}$ ) can be written as follows (Eq. 30) after analyzing velocities into two components:

$$
\left(\tau_{b x}, \tau_{b y}\right)=\frac{\rho_{w} g}{C_{z}^{2}}(\bar{u}, \bar{v}) \sqrt{\bar{u}^{2}+\bar{v}^{2}}
$$

\subsection{Heat and water quality transport governing equation}

Heat and water quality transport are governed by the advection diffusion equation which can be written in a general form as follows (Eq. 31): 


$$
\frac{\partial \phi}{\partial t}+\frac{\partial \bar{u} \phi}{\partial x}+\frac{\partial \bar{v} \phi}{\partial y}+\frac{\partial \hat{w} \phi}{\partial z}=\frac{\partial}{\partial x}\left[D_{x} \frac{\partial \phi}{\partial x}\right]+\frac{\partial}{\partial y}\left[D_{y} \frac{\partial \phi}{\partial y}\right]+\frac{\partial}{\partial z}\left[D_{z} \frac{\partial \phi}{\partial z}\right]+S_{\phi}
$$

where $\phi$ is the constituent concentration $\left(\mathrm{gm}^{-3}\right), S_{\phi}$ is the source/sink term of the constituent $\phi\left(\mathrm{gm}^{-3} \mathrm{sec}^{-1}\right)$, and $D_{x}, D_{y}$, and $D_{z}$ are the $x, y$, and z-diffusion coefficients (diffusivities) $\left(\mathrm{m}^{2} \mathrm{sec}^{-1}\right)$, respectively. Note that, in case of heat transport, $\phi$ is converted to a concentration of "heat", i.e., using the specific heat of water $\left(c_{p}\right)$ and water temperature $(T), \phi=\rho c_{p} T$.

\subsection{Numerical solution}

\section{The computational grid cells}

An equally spacing staggered grid distribution was used for all variables in the domain as shown in Fig. 3. Each cell was defined at the center by $i, j$, and $k(i=1,2, \ldots \ldots \ldots, i \max -2, i \max -1, i \max , j=1,2, \ldots \ldots \ldots$, jmax-2, jmax-1, jmax, and $k=1,2, \ldots \ldots \ldots, k \max -2, k \max -1, k \max )$. The parameters imax, jmax, and $k \max$ are the maximum value of $i, j$, and $k$ in the $x, y$, and $z$-direction, respectively. The domain was divided into computational cells. Some variables other than velocities were defined at the center of the cell, while others were defined at the sides of the cell. The variables $\rho, \phi$, and $H$ were defined at the center $(i, j, k)$, whereas $u, D_{x}, \tau_{x x}$, and $\tau_{y x}$ were defined at $(i+1 / 2, j, k)$ and $(i-1 / 2, j, k)$. The variables $v, D_{y}, \tau_{y y}$, and $\tau_{x y}$ were defined at $(i, j+1 / 2, k)$ and $(i, j-1 / 2, k)$, whereas $w, D_{z}$, and $v_{v}$ were defined at $(i, j, k+1 / 2)$ and $(i, j, k-1 / 2)$. Wherever a variable is required at a location other than its original defined location, linear interpolation based on its surrounding values is used.

\section{Numerical solution of the free surface equation}

The free water surface equation (Eq. 24) is solved by substituting the finite difference approximations of the $x$ and $y$-momentum equation (Eqs 32 and 33) into the free surface equation.

$$
\begin{aligned}
& \bar{u}_{i, j, k}^{n+1}=\bar{u}_{i, j, k}^{n}+\Delta t\left[F_{x}+g \cos \alpha \frac{\partial \eta}{\partial x}-\frac{g \cos \alpha}{\rho_{\circ}} \int_{\eta}^{z} \frac{\partial \rho}{\partial x} d z+\frac{1}{\rho_{\circ}} \frac{\partial\left(\tau_{x z}\right)}{\partial z}\right]_{i, j, k}^{n} \\
& \bar{v}_{i, j, k}^{n+1}=\bar{v}_{i, j, k}^{n}+\Delta t\left[F_{y}+g \cos \alpha \frac{\partial \eta}{\partial y}-\frac{g \cos \alpha}{\rho_{\circ}} \int_{\eta}^{z} \frac{\partial \rho}{\partial y} d z+\frac{1}{\rho_{\circ}} \frac{\partial\left(\tau_{y z}\right)}{\partial z}\right]_{i, j, k}^{n}
\end{aligned}
$$

where $F_{x}$ and $F_{y}$ are explicit operators that account for the advection, horizontal and vertical turbulent shear stresses, Coriolis, and gravitational acceleration component. The advective terms were then 
converted to finite difference equations by using the first-order upwind scheme. Also, the second-order central scheme was used for the horizontal and vertical diffusion terms.

Substituting $\bar{u}_{i, j, j}^{n+1}$ and $\bar{v}_{i, j, j}^{n+1}$ into free water surface equation for $\bar{u}$ and $\bar{v}$, respectively.

$$
\begin{aligned}
\frac{\partial \eta}{\partial t} & =\frac{\partial}{\partial x} \int_{\eta}^{h} \bar{u}_{i, j, k}^{n} d z+\left.\Delta t \frac{\partial}{\partial x} \int_{\eta}^{h} F_{x}\right|_{i, j, k} ^{n} d z+\left.\Delta t \frac{\partial}{\partial x} \int_{\eta}^{h} g \cos \alpha \frac{\partial \eta}{\partial x}\right|_{i, j} ^{n} d z-\left.\Delta t \frac{\partial}{\partial x} \int_{\eta}^{h} \frac{g \cos \alpha_{x}}{\rho_{\circ}} \int_{\eta}^{z} \frac{\partial \rho}{\partial x} d z\right|_{i, j, k} ^{n} d z \\
& +\left.\Delta t \frac{\partial}{\partial x} \int_{\eta}^{h} \frac{1}{\rho_{\circ}} \frac{\partial\left(\tau_{x z}\right)}{\partial z}\right|_{i, j, k} ^{n} d z+\frac{\partial}{\partial y} \int_{\eta}^{h} \bar{v}_{i, j, k}^{n} d z+\left.\Delta t \frac{\partial}{\partial y} \int_{\eta}^{h} F_{y}\right|_{i, j, k} ^{n} d z+\left.\Delta t \frac{\partial}{\partial y} \int_{\eta}^{h} g \cos \alpha \frac{\partial \eta}{\partial y}\right|_{i, j} ^{n} d z \\
& -\left.\Delta t \frac{\partial}{\partial y} \int_{\eta}^{h} \frac{g \cos \alpha_{y}}{\rho_{o}} \int_{\eta}^{z} \frac{\partial \rho}{\partial y} d z\right|_{i, j, k} ^{n} d z+\left.\Delta t \frac{\partial}{\partial y} \int_{\eta}^{h} \frac{1}{\rho_{0}} \frac{\partial\left(\tau_{y z}\right)}{\partial z}\right|_{i, j, k} ^{n} d z
\end{aligned}
$$

By defining new variables $\left.H_{r x}\right|_{i, j, k}$ and $\left.H_{r y}\right|_{i, j, k}$ for the water depth at the grid right face in the $x$ and $y$ direction, respectively,

$$
\left.H_{r x}\right|_{i, j, k}=\left.\frac{\left.H\right|_{i, j, k}+\left.H\right|_{i+1, j, k}}{2} \quad H_{r y}\right|_{i, j, k}=\frac{\left.H\right|_{i, j, k}+\left.H\right|_{i, j+1, k}}{2}
$$

and also, by using a backward difference for the unsteady term of Eq. 34,

$$
\begin{aligned}
& W \eta_{i-1, j}^{n}+N \eta_{i, j-1}^{n}+M \eta_{i, j}^{n}+S \eta_{i, j+1}^{n}+E \eta_{i+1, j}^{n}=R H S \\
& W=\left[\left.\frac{-\Delta t^{2} \Delta y g \cos \alpha}{\Delta x} \sum_{k=1}^{K} H\right|_{i-1, j}\right] \quad N=\left[\left.\frac{-\Delta t^{2} \Delta x g \cos \alpha}{\Delta y} \sum_{k=1}^{K} H\right|_{i, j-1}\right] \\
& M=\left[\Delta x \Delta y+\frac{\Delta t^{2} \Delta y g \cos \alpha}{\Delta x}\left(\left.\sum_{k=1}^{K} H\right|_{i, j}+\left.\sum_{k=1}^{K} H\right|_{i-1, j}\right)+\frac{\Delta t^{2} \Delta x g \cos \alpha}{\Delta y}\left(\left.\sum_{k=1}^{K} H\right|_{i, j}+\left.\sum_{k=1}^{K} H\right|_{i, j-1}\right)\right] \\
& S=\left[\left.\frac{-\Delta t^{2} \Delta x g \cos \alpha}{\Delta y} \sum_{k=1}^{K} H\right|_{i, j}\right] \quad E=\left[\left.\frac{-\Delta t^{2} \Delta y g \cos \alpha}{\Delta x} \sum_{k=1}^{K} H\right|_{i, j}\right] \\
& R H S=\Delta t \Delta y\left[\left.\sum_{k=1}^{K} \bar{u} H_{r x}\right|_{i, j}-\left.\sum_{k=1}^{K} \bar{u} H_{r x}\right|_{i-1, j}\right]+\Delta t \Delta x\left[\left.\sum_{k=1}^{K} \bar{v} H_{r y}\right|_{i, j}-\left.\sum_{k=1}^{K} \bar{v} H_{r y}\right|_{i, j-1}\right]+\Delta t^{2} \Delta y\left[\left.\sum_{k=1}^{K} F_{x} H_{r x}\right|_{i, j}-\left.\sum_{k=1}^{K} F_{x} H_{r x}\right|_{i-1, j}\right] \\
& +\Delta t^{2} \Delta x\left[\left.\sum_{k=1}^{K} F_{y} H_{r y}\right|_{i, j}-\left.\sum_{k=1}^{K} F_{y} H_{r y}\right|_{i, j-1}\right]-\frac{\Delta t^{2} \Delta y g \cos \alpha}{\rho_{o}} \sum_{k=1}^{K}\left(\left.\sum_{k=1}^{K} \frac{\partial \rho}{\partial x} H^{2}\right|_{i, j}-\left.\sum_{k=1}^{K} \frac{\partial \rho}{\partial x} H^{2}\right|_{i-1, j}\right) \\
& -\frac{\Delta t^{2} \Delta x g \cos \alpha}{\rho_{\circ}} \sum_{k=1}^{K}\left(\left.\sum_{k=1}^{K} \frac{\partial \rho}{\partial y} H^{2}\right|_{i, j}-\left.\sum_{k=1}^{K} \frac{\partial \rho}{\partial y} H^{2}\right|_{i, j-1}\right)+\frac{\Delta t^{2} \Delta y}{\rho_{\circ}}\left[\left(\tau_{b x}-\tau_{s x}\right)_{i, j}-\left(\tau_{b x}-\tau_{s x}\right)_{i-1, j}\right] \\
& +\frac{\Delta t^{2} \Delta x}{\rho_{\mathrm{o}}}\left[\left(\tau_{b y}-\tau_{s y}\right)_{i, j}-\left(\tau_{b y}-\tau_{s y}\right)_{i, j-1}\right]+\Delta x \Delta y \eta_{i, j}^{n-1}
\end{aligned}
$$

where $K$ is the bottom cell $k$ value, $k b(i, j)$. 
Applying Eq. 36 leads to a system of simultaneous linear algebraic equations. This system has a matrix form of penta-diagonal coefficients and can be solved by any suitable iterative or direct method. The present 3D model used a method called line-by-line (Patankar, 1980).

\section{Numerical solution of the momentum equations}

The $x$-momentum equation (Eq. 21) is solved by splitting the horizontal momentum equations into two equations in two stages at each model time step. One model stage is treated explicitly, while the other stage is treated implicitly. For the $x$-momentum equation,

$$
\begin{gathered}
\frac{\partial \bar{u}}{\partial t}=-\bar{u} \frac{\partial \bar{u}}{\partial x}-\bar{v} \frac{\partial \bar{u}}{\partial y}-\bar{w} \frac{\partial \bar{u}}{\partial z}+g \cos \alpha \frac{\partial \eta}{\partial x}-\frac{g \cos \alpha}{\rho_{\circ}} \int_{\eta}^{z} \frac{\partial \rho}{\partial x} d z+f \bar{v}+g \sin \alpha \\
+\frac{1}{\rho_{\circ}} \frac{\partial\left(\tau_{x x}\right)}{\partial x}+\frac{1}{\rho_{\circ}} \frac{\partial\left(\tau_{x y}\right)}{\partial y}+\frac{1}{\rho_{\circ}} \frac{\partial\left(\tau_{s x}+\tau_{b x}\right)}{\partial z} \\
\frac{\partial \bar{u}}{\partial t}=\frac{1}{\rho_{\circ}} \frac{\partial\left(\tau_{x z}\right)}{\partial z}
\end{gathered}
$$

In the first stage, Eq. 39 was solved explicitly as follows:

$$
\bar{u}_{i, j, k}^{*}=\bar{u}_{i, j, k}^{n}+\Delta t\left[\begin{array}{l}
-\bar{u} \frac{\partial \bar{u}}{\partial x}-\bar{v} \frac{\partial u}{\partial y}-\bar{w} \frac{\partial u}{\partial z}+g \cos \alpha \frac{\partial \eta}{\partial x}-\frac{g \cos \alpha}{\rho_{\circ}} \int_{\eta}^{z} \frac{\partial \rho}{\partial x} d z+f \bar{v}+g \sin \alpha \\
+\frac{1}{\rho_{\circ}} \frac{\partial\left(\tau_{x x}\right)}{\partial x}+\frac{1}{\rho_{\circ}} \frac{\partial\left(\tau_{x y}\right)}{\partial y}+\frac{1}{\rho_{\circ}} \frac{\partial\left(\tau_{s x}+\tau_{b x}\right)}{\partial z}
\end{array}\right]_{i, j, k}^{n}
$$

where $\bar{u}_{i, j, k}^{*}$ represents the value of $\bar{u}_{i, j, k}$ at $t=t+\Delta t$.

By defining [[a1,a2]] and $((a 1, a 2))$ to be the maximum and minimum value of (a1 and $a 2)$, respectively, the final explicit finite difference equation of $\bar{u}_{i, j, k}^{*}$ : 


$$
\begin{aligned}
\bar{u}_{i, j, k}^{*}= & \bar{u}_{i, j, k}^{n}-\frac{\Delta t}{\Delta x}\left[\bar{u}_{i, j, k}\left[\left[\bar{u}_{i, j, k}, 0\right]\right]-\bar{u}_{i, j, k}\left(\left(\bar{u}_{i, j, k}, 0\right)\right)-\bar{u}_{i-1, j, k}\left[\left[\bar{u}_{i, j, k}, 0\right]\right]+\bar{u}_{i+1, j, k}\left(\left(\bar{u}_{i, j, k}, 0\right)\right)\right]^{n} \\
& -\frac{\Delta t}{\Delta y}\left[\bar{u}_{i, j, k}\left[\left[\bar{v}_{i, j, k}, 0\right]\right]-\bar{u}_{i, j, k}\left(\left(\bar{v}_{i, j, k}, 0\right)\right)-\bar{u}_{i, j-1, k}\left[\left[\bar{v}_{i, j, k}, 0\right]\right]+\bar{u}_{i, j+1, k}\left(\left(\bar{v}_{i, j, k}, 0\right)\right)\right]^{n} \\
& -\frac{\Delta t}{\Delta z}\left[\bar{u}_{i, j, k}\left[\left[\bar{w}_{i, j, k}, 0\right]\right]-\bar{u}_{i, j, k}\left(\left(\bar{w}_{i, j, k}, 0\right)\right)-\bar{u}_{i, j-1, k}\left[\left[\bar{w}_{i, j, k}, 0\right]\right]+\bar{u}_{i, j+1, k}\left(\left(\bar{w}_{i, j, k}, 0\right)\right)\right]^{n} \\
& +\frac{\Delta t g \cos \alpha}{\Delta x}\left(\eta_{i+1, j, k}^{n}-\eta_{i, j, k}^{n}\right)-\left.\frac{\Delta \operatorname{tg} \cos \alpha}{\rho_{\mathrm{o}} \Delta x} \sum_{k=1}^{K}\left(\rho_{i+1, j, k}^{n}-\rho_{i, j, k}^{n}\right) H_{r x}\right|_{i, j, k} ^{n}+\Delta t f \bar{v}_{i, j, k}^{n} \\
& +\Delta t g \sin \alpha+\frac{\Delta t v_{h}}{\Delta x_{i, j, k}}\left[\frac{\bar{u}_{i+1, j, k}-\bar{u}_{i, j, k}}{\Delta x_{i+1 / 2, j, k}}-\frac{\bar{u}_{i, j, k}-\bar{u}_{i-1, j, k}}{\Delta x_{i-1 / 2, j, k}}\right]^{n} \\
& +\frac{\Delta t v_{h}}{\Delta y_{i, j, k}}\left[\frac{\bar{u}_{i, j+1, k}-\bar{u}_{i, j, k}}{\Delta y_{i, j+1 / 2, k}}-\frac{\bar{u}_{i, j, k}-\bar{u}_{i, j-1, k}}{\Delta y_{i, j-1 / 2, k}}\right]^{n} \\
& +\frac{\Delta t}{\rho_{\mathrm{o}} \Delta z}\left[\left(\tau_{s x}+\tau_{b x}\right)_{i, j, k+1 / 2}-\left(\tau_{s x}+\tau_{b x}\right)_{i, j, k-1 / 2}\right]^{n}
\end{aligned}
$$

Now, $\bar{u}_{i, j, k}^{*}$ is calculated for each grid and will be used to calculate $\bar{u}_{i, j, k}^{n+1}$ by solving the second equation implicitly. In the second stage, Eq. 40 was solved by using a fully implicit finite difference technique for the vertical diffusion term as follows:

$$
\begin{gathered}
\frac{\partial \bar{u}}{\partial t}=\frac{\partial}{\partial z}\left[v_{v} \frac{\partial \bar{u}}{\partial z}\right] \\
\frac{\bar{u}_{i, j, k}^{n+1}-\bar{u}_{i, j, k}^{*}}{\Delta t}=\frac{1}{\Delta z_{i, j, k}}\left[v_{v_{i, j, k}} \frac{\bar{u}_{i, j, k+1}^{n+1}-\bar{u}_{i, j, k}^{n+1}}{\Delta z_{i, j, k+1 / 2, k}}-v_{v_{i, j, k-1}} \frac{\bar{u}_{i, j, k}^{n+1}-\bar{u}_{i, j, k-1}^{n+1}}{\Delta z_{i, j, k-1 / 2, k}}\right]
\end{gathered}
$$

Equation 44 was simplified and then arranged to become:

$$
\begin{array}{r}
G \bar{u}_{i, j, k-1}^{n+1}+F \bar{u}_{i, j, k}^{n+1}+Q \bar{u}_{i, j, k+1}^{n+1}=\bar{u}_{i, j, k}^{*} \\
G=\left[-\frac{\Delta t v_{v_{i, j, k-1}}}{\Delta z_{i, j, k} \Delta z_{i, j, k-1 / 2}}\right] \\
F=\left[1+\frac{\Delta t v_{v_{i, j, k-1}}}{\Delta z_{i, j, k} \Delta z_{i, j, k+1 / 2}}+\frac{\Delta t v_{v_{i, j, k}}}{\Delta z_{i, j, k} \Delta z_{i, j, k-1 / 2}}\right] \\
Q=\left[-\frac{\Delta t v_{v_{i, j, k}}}{\Delta z_{i, j, k} \Delta z_{i, j, k+1 / 2}}\right]
\end{array}
$$

A system of linear algebraic equations for each water column in the domain is solved by using Thomas algorithm (Patankar, 1980). In addition, a similar procedure was used to solve the $y$-momentum equation (Eq. 22). Note that, the present model has an equally spacing grid. However, the grid spacing at the cells 
faces was used and denoted by $i+1 / 2, j+1 / 2$, and $k+1 / 2$. This was done just with diffusion terms to explain the discretization procedure.

\section{Numerical solution of the continuity equation}

After calculating $\bar{u}_{i, j, k}^{n+1}$ and $\bar{v}_{i, j, k}^{n+1}$, the vertical velocity component $\bar{w}_{i, j, k}^{n+1}$ can be calculated from the continuity equation (Eq. 6) by implementing the cell by cell calculations as follows:

$$
\begin{gathered}
\frac{\bar{u}_{i, j, k}^{n+1}-\bar{u}_{i-1, j, k}^{n+1}}{\Delta x}+\frac{\bar{v}_{i, j, k}^{n+1}-\bar{v}_{i, j-1, k}^{n+1}}{\Delta y}+\frac{\bar{w}_{i, j, k}^{n+1}-\bar{w}_{i, j, k-1}^{n+1}}{\Delta z}=0 \\
\bar{w}_{i, j, k-1}^{n+1}=\bar{w}_{i, j, k}^{n+1}+\frac{\bar{u}_{i, j, k}^{n+1}-\bar{u}_{i-1, j, k}^{n+1}}{\Delta x} \Delta z+\frac{\bar{v}_{i, j, k}^{n+1}-\bar{v}_{i, j-1, k}^{n+1}}{\Delta y} \Delta z
\end{gathered}
$$

Numerical solution of heat and water quality transport equation

The water quality transport equation (Eq. 31) is also split into two equations in two stages and solved at each model time step. The solution procedure is similar to the solution of the momentum equations in which the source/sink term of Eq. 31 is treated explicitly. Because the solution of transport equation is very critical at the regions where there is a concentration discontinuity such as at the thermocline level during the stratification period in lakes and reservoirs, higher-order schemes are recommended to use for the horizontal advective terms (Cole \& Wells, 2015; Edinger, 2001; Kowalik \& Murty, 1993; Leonard, 1979, 1991; Neumann, Simunek, \& Cook, 2011). Thus, QUICKEST scheme has been adopted and formulated based on Kowalik and Murty (1993).

$$
\begin{gathered}
\frac{\partial \phi}{\partial t}+\frac{\partial \bar{u} \phi}{\partial x}+\frac{\partial \hat{v} \phi}{\partial y}+\frac{\partial \bar{w} \phi}{\partial z}=\frac{\partial}{\partial x}\left[D_{x} \frac{\partial \phi}{\partial x}\right]+\frac{\partial}{\partial y}\left[D_{y} \frac{\partial \phi}{\partial y}\right]+S_{\phi} \\
\frac{\partial \phi}{\partial t}=\frac{\partial}{\partial z}\left[D_{z} \frac{\partial \phi}{\partial z}\right]
\end{gathered}
$$

The finite difference formulation for the first stage

$$
\begin{aligned}
\left.a d v\right|_{i, j, k} ^{n}= & \left(q_{p x}+q_{n x}\right)\left[\frac{1}{2}\left(\phi_{i+1, j, k}-\phi_{i-1, j, k}\right)-\frac{1}{2} \Delta t\left(q_{p x}+q_{n x}\right)\left(\phi_{i+1, j, k}+\phi_{i-1, j, k}-2 \phi_{i, j, k}\right)\right] \\
& +\frac{1}{6} q_{p x}\left(1-q_{p x}{ }^{2} \Delta t^{2}\right)\left(\phi_{i-2, j, k}-3 \phi_{i-1, j, k}+3 \phi_{i, j, k}-\phi_{i+1, j, k}\right) \\
& +\frac{1}{6} q_{n x}\left(1-q_{n x}{ }^{2} \Delta t^{2}\right)\left(\phi_{i-1, j, k}-3 \phi_{i, j, k}+3 \phi_{i+1, j, k}-\phi_{i+2, j, k}\right) \\
& +\left(q_{p y}+q_{n y}\right)\left[\frac{1}{2}\left(\phi_{i, j+1, k}-\phi_{i, j-1, k}\right)-\frac{1}{2} \Delta t\left(q_{p y}+q_{n y}\right)\left(\phi_{i, j+1, k}+\phi_{i, j-1, k}-2 \phi_{i, j, k}\right)\right] \\
& +\frac{1}{6} q_{p y}\left(1-q_{p y}{ }^{2} \Delta t^{2}\right)\left(\phi_{i, j-2, k}-3 \phi_{i, j-1, k}+3 \phi_{i, j, k}-\phi_{i, j+1, k}\right) \\
& +\frac{1}{6} q_{n y}\left(1-q_{n y}{ }^{2} \Delta t^{2}\right)\left(\phi_{i, j-1, k}-3 \phi_{i, j, k}+3 \phi_{i, j+1, k}-\phi_{i, j+2, k}\right)
\end{aligned}
$$




$$
\begin{aligned}
& +\left(q_{p z}+q_{n z}\right)\left[\frac{1}{2}\left(\phi_{i, j, k+1}-\phi_{i, j, k-1}\right)-\frac{1}{2} \Delta t\left(q_{p z}+q_{n z}\right)\left(\phi_{i, j, k+1}+\phi_{i, j, k-1}-2 \phi_{i, j, k}\right)\right] \\
& +\frac{1}{6} q_{p z}\left(1-q_{p z}^{2} \Delta t^{2}\right)\left(\phi_{i, j, k-2}-3 \phi_{i, j, k-1}+3 \phi_{i, j, k}-\phi_{i, j, k+1}\right) \\
& +\frac{1}{6} q_{n z}\left(1-q_{n z}^{2} \Delta t^{2}\right)\left(\phi_{i, j, k-1}-3 \phi_{i, j, k}+3 \phi_{i, j, k+1}-\phi_{i, j, k+2}\right)
\end{aligned}
$$

where:

$$
\begin{gathered}
q_{p x}=\frac{1}{2}\left[q_{x}+\left|q_{x}\right|\right] \quad q_{n x}=\frac{1}{2}\left[q_{x}-\left|q_{x}\right|\right] \\
q_{p y}=\frac{1}{2}\left[q_{y}+\left|q_{y}\right|\right] \quad q_{n y}=\frac{1}{2}\left[q_{y}-\left|q_{y}\right|\right] \\
q_{p z}=\frac{1}{2}\left[q_{z}+\left|q_{z}\right|\right] \quad q_{n z}=\frac{1}{2}\left[q_{z}-\left|q_{z}\right|\right] \\
\left.\operatorname{diff}\right|_{i, j, k} ^{n}=\frac{D_{x}}{\Delta x_{i, j, k}}\left[\frac{\phi_{i+1, j, k}-\phi_{i, j, k}}{\Delta x_{i+1 / 2, j, k}}-\frac{\phi_{i, j, k}-\phi_{i-1, j, k}}{\Delta x_{i-1 / 2, j, k}}\right]^{n}+\frac{D_{y}}{\Delta y_{i, j, k}}\left[\frac{\phi_{i, j+1, k}-\phi_{i, j, k}}{\Delta y_{i, j+1 / 2, k}}-\frac{\phi_{i, j, k}-\phi_{i, j-1, k}}{\Delta y_{i, j-1 / 2, k}}\right]^{n} \\
\left.\phi\right|_{i, j, k} ^{*}=\left.\phi\right|_{i, j, k} ^{n}+\Delta t\left(-\left.a d v\right|_{i, j, k} ^{n}+\left.\operatorname{diff}\right|_{i, j, k} ^{n}+\left.S_{\phi}\right|_{i, j, k} ^{n}\right)
\end{gathered}
$$

Also, the finite difference formulation of the second stage ends with:

$$
\begin{gathered}
U \phi_{i, j, k-1}^{n+1}+C \phi_{i, j, k}^{n+1}+D \phi_{i, j, k+1}^{n+1}=\phi_{i, j, k}^{*} \\
U=\left[-\frac{\Delta t D_{z_{i, j, k-1}}}{\Delta z_{i, j, k} \Delta z_{i, j, k-1 / 2}}\right] \\
C=\left[1+\frac{\Delta t D_{z_{i, j, k}}}{\Delta z_{i, j, k} \Delta z_{i, j, k+1 / 2}}+\frac{\Delta t D_{z_{i, j, k-1}}}{\Delta z_{i, j, k} \Delta z_{i, j, k-1 / 2}}\right] \\
D=\left[-\frac{\Delta t D_{z_{i, j, k}}}{\Delta z_{i, j, k} \Delta z_{i, j, k+1 / 2}}\right]
\end{gathered}
$$

Numerical solution of the linear algebraic equations

The final solution of the free surface equation, momentum equations, and transport equation was obtained by solving a system of linear algebraic equations. Each system can be arranged in a matrix form (Eq. 56):

$$
[\mathbf{A}][\boldsymbol{X}]=[\mathbf{B}]
$$

Where $[\mathbf{A}]$ is the coefficients matrix which is diagonally predominant, $[\boldsymbol{X}]$ is the unknown column matrix, and $[\mathbf{B}]$ is the right hand side coefficients column matrix. 
There are several methods for solving a set of linear algebraic equations; some are more suitable than others based on the accuracy and numerical efficiency. Therefore, any suitable method could be used here. The form of the system of linear algebraic equations plays a major role in choosing the solution method. The solution of the momentums and transport equation ends with a coefficients matrix in a tridiagonal form where the main diagonal elements are positive and the two off-diagonal elements are negative. The most convenient direct method that is widely and easily used in solving a system of linear equations in which [A] is a tri-diagonal matrix is the Thomas algorithm (Patankar, 1980), also called the tri-diagonal matrix algorithm. We employed a time splitting technique in solving the equations of momentum and transport since these equations lead to a simple tri-diagonal matrix which is diagonally predominant and can be easily solved by Thomas algorithm.

For instance, Eq. 54 is written at each of the unknown nodal points along the water column from top to bottom to form a system of simultaneous linear algebraic equations for each water column. The general matrix form of this system can be written as follows:

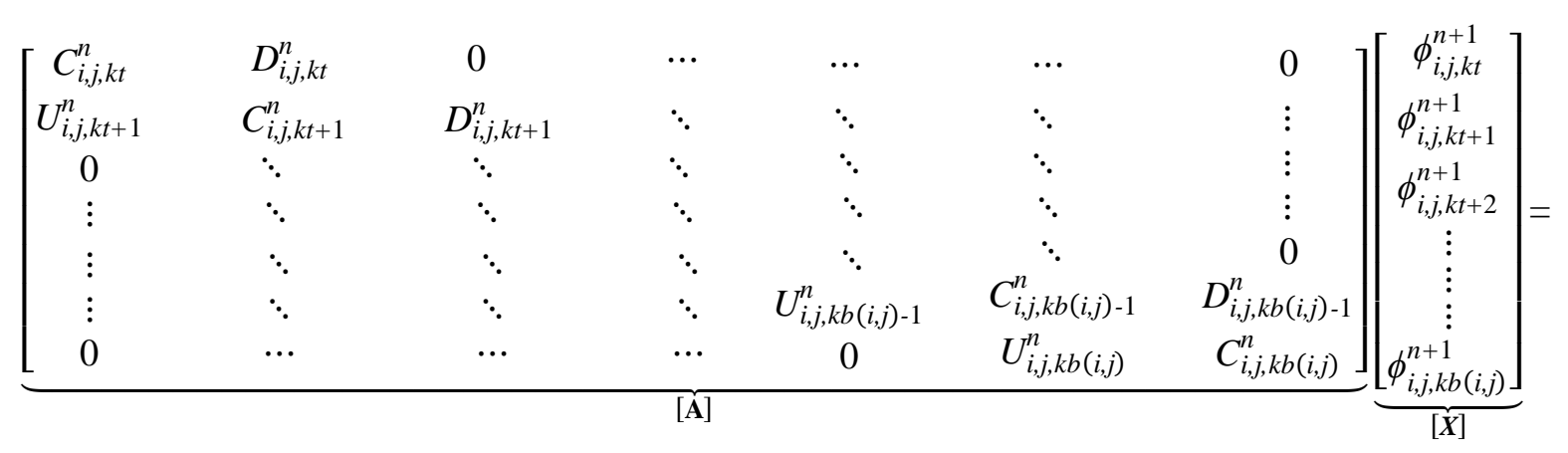

$\underbrace{\left[\begin{array}{c}R_{i, j, k t}^{n} \\ R_{i, j, k t+1}^{n} \\ R_{i, j, k t+2}^{n} \\ \vdots \\ \vdots \\ R_{i, j, k b(i, j)}^{n}\end{array}\right]}_{[\mathbf{B}]}$

where $R$ is the solution of the first stage.

Thomas algorithm for solving this system is as follows:

- Calculate $\gamma_{i, j, k t}$ and $\alpha_{i, j, k t}$ :

$$
\gamma_{i, j, k t}=\frac{D}{C} \quad \alpha_{i, j, k t}=\frac{R}{C}
$$

- Calculate $\gamma_{i, j, k}$ and $\alpha_{i, j, k}$ for $k=k t+1, k t+2, \ldots \ldots \ldots, k b(i, j)$ : 


$$
\begin{aligned}
\gamma_{i, j, k} & =\frac{D}{C-\left(U^{*} \gamma_{i, j, k-1}\right)} \\
\alpha_{i, j, k} & =\frac{R-\left(U^{*} \alpha_{i, j, k-1}\right)}{C-\left(U^{*} \gamma_{i, j, k-1}\right)}
\end{aligned}
$$

- $\quad$ Set $\phi_{i, j, k b(i, j)}^{n+1}=\alpha_{i, j, k b(i, j)}$

- Calculate $\phi_{i, j, k}^{n+1}$ for $k=k b(i, j)-1, k b(i, j)-2, \ldots \ldots \ldots, k t$ :

$$
\phi_{i, j, k}^{n+1}=\alpha_{i, j, k}-\left(\gamma_{i, j, k} * \phi_{i, j, k+1}^{n+1}\right)
$$

where $k t$ and $k b$ are the top and bottom cells, respectively.

While the solution of the momentum and transport equations results in a tri-diagonal matrix coefficients matrix, the free surface equation solution has a penta-diagonal coefficients matrix. Using direct methods for solving the simultaneous linear algebraic equations are much more complicated and require rather large amount of computer storage and time; therefore, iterative methods could be used such as the conjugate gradient method. However, direct methods are more accurate than iterative methods. In the present model, the line-by-line method, a combination of the Thomas algorithm and Gauss-Sidel method, has been used (Patankar, 1980) to solve the system of simultaneous linear algebraic equations generated from the numerical solution of free surface equation.

Equation 36 is written for each unknown water surface elevations (sweeping in either direction and ordering line-by-line) to form a system of simultaneous linear algebraic equations. If we wrote this system in a matrix form for a rectangular domain, the matrix [A] would have penta-diagonal coefficients. In the line-by-line method, one direction of the system is assumed unknown, and the other direction is considered known taken from the latest values. Therefore, the system of the penta-diagonal coefficients matrix in Eq. 36 was converted to a system of tri-diagonal coefficients matrices (Eq. 59), which are easily solved by Thomas algorithm.

$$
W \eta_{i-1, j}^{n}+M \eta_{i, j}^{n}+E \eta_{i+1, j}^{n}=R H S-N \eta_{i, j-1}^{n-1}-S \eta_{i, j+1}^{n-1}
$$

The present model sweeps in the $x$-direction (main stream direction), line-by-line, to transfer flow along the main flow direction. Another advantage of using the line-by-line method other than the low computational cost is that the method helps with irregular boundaries in which each row of neighbouring unknowns is solved separately by Thomas algorithm.

\section{Results and discussion}

The analytical verification of the model hydrodynamics was done by comparing model predictions to known analytical exact solutions test cases. 


\subsection{Test 1: Free surface seiching in a closed rectangular basin}

This test was done in a similar way to that test recommended by Wang, Roache, Schmalz, Jia, \& Smith (2009). A closed rectangular basin was subjected to an initial vertical displacement in which the free surface wave has a profile of a half cosine in the longitudinal direction as shown in Fig. 4. The basin dimensions are: $L=38000 \mathrm{~m}, B=6000 \mathrm{~m}$, and $H=12 \mathrm{~m}$. The initial vertical amplitude at the left and right boundaries of the basin, $\eta_{\mathrm{o}}$, is $25 \mathrm{~cm}$. Thus, after releasing the system from the rest, the oscillated wave starts and continues with time. If there were frictional resistance, the wave is damped and eventually the system goes to the rest. Also, we will make the following assumptions:

- At the closed boundaries, the longitudinal and lateral velocities are zero.

- The advection terms, diffusion terms, and boundary shear stresses are neglected.

- The fluid is inviscid and has a constant density, $\rho=1000 \mathrm{kgm}^{-3}$.

- The Coriolis force is neglected $(f=0)$.

- There are no sources/sinks of fluid mass.

- Boussinesq approximations are valid and the pressure is hydrostatic.

Based on the above assumptions, the one-dimensional governing equations are

$$
\begin{aligned}
& \frac{\partial \eta}{\partial t}+\frac{\partial u H}{\partial x}=0 \\
& \frac{\partial u}{\partial t}+g \frac{\partial \eta}{\partial x}=0
\end{aligned}
$$

The analytical solution was given by Eliason and Bourgeois (1997) (Eq. 61):

$$
\begin{gathered}
\eta_{(x, t)}=\eta_{\circ} \cos \left(\frac{\pi x}{L}\right) \cos \left(\frac{\pi \sqrt{g H}}{L} t\right) \\
u_{(x, t)}=\frac{\eta_{\circ} \sqrt{g H}}{H} \sin \left(\frac{\pi x}{L}\right) \sin \left(\frac{\pi \sqrt{g H}}{L} t\right)
\end{gathered}
$$

Subjected to initial conditions (Eq. 62):

$$
\eta_{(x, 0)}=-0.25 \times \cos \left(\frac{\pi x}{L}\right) \quad u=v=w=0
$$

and boundary conditions of $(u=v=w=0)$ at the closed boundaries in addition to 


$$
\begin{aligned}
& \frac{\partial v}{\partial x}=\frac{\partial \eta}{\partial x}=0 \\
& \frac{\partial u}{\partial y}=\frac{\partial \eta}{\partial y}=0 \\
& \frac{\partial u}{\partial z}=\frac{\partial v}{\partial z}=0
\end{aligned}
$$

The domain was divided into 1575 computational cells of $(21 \times 5 \times 15, x \times y \times z)$, in which $k t=3$ (the location of the surface layer is at the vertical layer of $k=3$ ) and internal cells of 684. The size of the computational cells is $\Delta x=\Delta y=2000 \mathrm{~m}$ and $\Delta z=1 \mathrm{~m}$.

The advection and diffusion terms, top and bottom shear stresses, and Coriolis force were set to zero in the model to agree with the analytical solution. To maintain a stable solution, the time step should be satisfied by the gravity courant number stability condition, $(\sqrt{g H} \Delta t / \Delta x) \leq 1$. Thus, we used time steps of $\Delta t=100,70,50$, and 5 sec to guarantee the stability and for comparison with analytical solution.

A comparison in the water level $(\eta)$ and longitudinal velocity $(u)$ between the model results and the analytical solution for the seiching basin is shown in Figs 5, 6, and 7. The comparison was done near the right and left boundary of the basin where the wave amplitude is high enough to see. The distance between the selected left location and the nearest boundary is equal to the distance between the selected right location and its nearest boundary, i.e. symmetric locations. This helps to ensure that if the solution were correct, the two waves at these locations would have the same magnitude at the same time of simulation but in opposite directions. The results show good agreement with the analytical solution even though there is damping of the numerical solution. The damping arises from using an implicit technique in the solution of the free surface equation (Vreugdenhil, 1989). The implicit scheme helps eliminate the celerity stability condition (Wells, 2002), however it has wave damping. Figure 8 shows the effect of time step on wave damping. Even though the criterion for the time step for stability was satisfied, the numerical code still had wave dampening for the higher time steps. But by reducing the time step below that required for stability, the damping decreased significantly. This agrees with Vreugdenhil (1989) who showed that "the time step is the major factor influencing the accuracy". The numerical solution will agree with the analytical solution without phase lag for any time step within the stability region. Wells (2002) showed that running the model with a high time step that may be numerically stable does not guarantee numerical accuracy.

Furthermore, Fig. 9 shows the model predictions of water level by using two spatial resolutions $(\Delta x=$ $\Delta y=2000 \mathrm{~m}$ and $\Delta x=\Delta y=1000 \mathrm{~m}$ ) and same time step in which the model is stable for both resolutions. Both results of the model are almost the same, indicating that the model produces similar predictions with similar numerical behaviour under stable conditions. 


\subsection{Test 2: Free water surface response to wind-induced flow in a closed rectangular basin}

To evaluate the influence of wind shear stress $\tau_{s}$ on the numerical solution in test case 1 , we added the surface shear stress to $x$-momentum equation that governs the seiching basin. Then, the governing momentum equation of this test is:

$$
\frac{\partial u}{\partial t}+g \frac{\partial \eta}{\partial x}=\frac{\tau_{s}}{\rho_{\mathrm{o}} H}
$$

If we considered $x=0$ at the center of the basin as shown in Fig. 10, in which $L=2 b$, and suddenly constant wind starts hitting the flat water surface, $\eta=0$, in the positive $x$-direction and continues blowing with time, the analytical solution for the water elevation was given by Wells (2002) (Eq. 65), where $u_{*}$ is the surface shear velocity.

$\eta_{(x, t)}=\frac{u_{*}^{2}}{g H} x-\frac{8 b u_{*}^{2}}{\pi^{2} g H}\left[\cos \left(\frac{\pi \sqrt{g H} t}{2 b}\right) \sin \left(\frac{\pi x}{2 b}\right)-\frac{1}{9} \cos \left(\frac{3 \pi \sqrt{g H} t}{2 b}\right) \sin \left(\frac{3 \pi x}{2 b}\right)+\frac{1}{25} \cos \left(\frac{5 \pi \sqrt{g H} t}{2 b}\right) \sin \left(\frac{5 \pi x}{2 b}\right)-\cdots\right]$

The code of test case 1 was run with constant wind of $2 \mathrm{msec}^{-1}$ at $10 \mathrm{~m}$ height above the water surface at $t=0$. A comparison in the water level $(\eta)$ between the model results and the analytical solution near the left boundary of the seiching basin is shown in Fig. 11.

The model showed good results in following the surface wave signal of the analytical solution with a performance similar to that in test case 1.

Therefore, we could say that under the effect of wind there is no extra damping to the surface wave compared to the case where there is no wind. Figure 12 shows the wind effect on the water surface level

upstream and downstream of the basin. In this case there are waves of opposite directions at both left and right end similar to those of test case 1 , but here the upstream wave has positive amplitude which is greater than the negative amplitude of the downstream wave, implying the water surface has a positive slope in the wind direction. Thus, this confirms the good agreement of the model to the basic theories of transporting the one-dimensional water surface waves under the influence of wind.

\subsection{Test 3: Velocity profile response to the wind induced flow in a closed rectangular basin}

The surface shear stresses due to the wind blowing on the waterbody transfer vertically resulting in a velocity profile (see Fig. 13) in which the water surface flows in the direction of the wind downstream and then it hits the boundary and circulates back upstream through the bottom layers. Different models are available to represent the analytical velocity profile. One of these models is a model developed by Hansen (1975). The analytical solution is: 


$$
\frac{u}{u_{*}}=6.65\left[1-\operatorname{erf}\left(\frac{z}{0.267 u * t}\right)\right]
$$

where $u$ is the longitudinal velocity over time at a depth of $z$ below the water surface.

Since the solution is based on assuming there is a balance between the rate of change of the longitudinal velocity and its vertical diffusion only, we need to run the code for a short period of time when the change in the water surface level can be considered negligible to agree with the analytical solution. Also, we need to turn off the horizontal advection, horizontal diffusion, and Coriolis force. Using a constant vertical eddy viscosity over depth $v_{t}=u_{*}^{2} t / 28$ (Wells, 2002) and wind of $10 \mathrm{msec}^{-1}$ in the positive $x$-direction, the code was run for 1000 sec. Figure 14 shows the computed and analytical velocity profile under the effect of wind induced flow in the middle of the seiching basin where the effect of circulation and boundaries are negligible. The model agreement with the analytical solution is important because in lakes and reservoirs, wind induced currents determine the vertical mixed thermal structure. The vertical mixed thermal structure then can affect water quality including algae growth dynamics which through self-shading can affect the density regime. Hence, it is necessary to have a 3D model that solves the hydrodynamic equations at the same time level as the water quality equations.

\subsection{Test 4: A case study}

Thermal stratification in Lake Chaplain, Washington, USA, was modeled using the three-dimensional model. Lake Chaplain (see Fig. 15) is a reservoir located at a latitude and longitude of 47.9592309 and 121.8447615, respectively, with an approximate elevation of $198 \mathrm{~m}$ above sea level. The inflow to the lake is a diverted flow from Spada Lake. There are two withdrawal outflows from Lake Chaplain; one of them is a pipe withdrawal flow from the lake north end toward a paper mill, the other is a drinking water withdrawal flow through an outflow structure at the dam which is at the south end of the lake. Each of the withdrawals exercised the selective withdrawal algorithm of the 3D model which based on that from Cole and Wells (2015).

The physical domain of the lake was divided into computational grid cells as shown in Fig. 16, which is a contour plot top view of the lake surface. Spatial resolution of $\Delta x=220 \mathrm{~m}, \Delta y=110 \mathrm{~m}$, and $\Delta z=1 \mathrm{~m}$ was used. Based on the setup in Fig. 16, $\theta_{2}$ is 5.49 radians. Initially, the water surface level was at an elevation of $195.73 \mathrm{~m}$, based on available data (Aug10, 2007 - Oct11, 2007).

The inflow to the domain enters the main waterbody at a water depth of $15 \mathrm{~m}$. The outflow to the paper mill that is located at a center-line elevation of $172 \mathrm{~m}$ at the model grid point $i=6$ and $j=11$. The other outflow to a water treatment plant is located at an elevation of $184.8 \mathrm{~m}$ at grid point $i=16$ and $j=7$, the dam location. A time series of the boundary inflow and outflow are shown in Figs 17, 18 , and 19. In addition, variable temperature inflow was used over the period of simulation (see Fig. 20). Wind 
magnitude and direction, air temperature, dew point, and cloud cover are necessary meteorological inputs for the water heat budget and surface heat exchange. All field data and meteorological data were described in Annear, Berger, and Wells (2008). Also, the model calculated short wave radiation internally based on an algorithm described by EPA (1971).

The model separates the vertical turbulent kinematic viscosity, $v_{v}$, into two parts (turbulent plus molecular viscosity), i.e.,

$$
v_{v}=v_{t}+v_{m}
$$

where $v_{t}$ is the vertical turbulent kinematic viscosity and $v_{m}$ is the minimum turbulent kinematic viscosity or molecular viscosity, assumed approximately $10^{-6} \mathrm{~m}^{2} \mathrm{sec}^{-1}$.

Hence, as turbulence decays at boundaries or strong stratification, the turbulent shear stresses go to zero.

The vertical turbulent kinematic viscosity under neutral stability conditions, $v_{t o}$, is calculated based on the Von Karman formula (Eq. 68) (Cole \& Buchak, 1995; Edinger, 2001):

$$
v_{t \circ}=K \frac{l^{2}}{2} \sqrt{\left[\frac{\partial \bar{u}}{\partial z}\right]^{2}+\left[\frac{\partial \bar{v}}{\partial z}\right]^{2}}
$$

where $\mathrm{K}$ is Von Karman constant, $0.4, l$ is the vertical mixing length scale, chosen to be the vertical increment $\Delta z$ (Cole \& Buchak, 1995).

To account for the turbulent mixing intensity due to vertical stratification, the vertical turbulent mixing is modified by employing the Richardson number criterion for vertical transport of momentum. The following modification (Eq. 69) was proposed by Leendertse and Liu (1975):

$$
v_{t}=v_{t_{\circ}} \exp (-1.5 \mathrm{Ri})
$$

where $\mathrm{Ri}$ is the Richardson number.

For the three-dimensional case, the Richardson number can be written as follows (Eq. 70) (Sheng \& Butler, 1982):

$$
\mathrm{Ri}=\frac{g \frac{\partial \rho}{\partial z}}{\rho_{\mathrm{o}}\left[\left(\frac{\partial \bar{u}}{\partial z}\right)^{2}+\left(\frac{\partial \bar{v}}{\partial z}\right)^{2}\right]}
$$

The vertical turbulent eddy diffusivity (vertical diffusion coefficient), $D_{z}$, is calculated from the vertical turbulent kinematic viscosity, $v_{v}$, using the Reynold's analogy (Eq. 71) (Cole \& Wells, 2015):

$$
D_{z}=0.14 v_{v}
$$

Additionally, the horizontal turbulent eddy viscosity, $v_{h}$, describes the transport of momentums horizontally between control volumes and was assumed a constant value of $1 \mathrm{~m}^{2} \mathrm{sec}^{-1}$. The horizontal 
turbulent eddy diffusivity in $x$ and $y$-direction ( $D_{x}$ and $D_{y}$, respectively) was related to the grid resolution based on Okubo (1971) as follows (Eqs 72 and 73) (Edinger 2001; Cole \& Wells 2015):

$$
\begin{aligned}
& D_{x}=5.85 \times 10^{-1.1 \Delta x} \\
& D_{y}=5.85 \times 10^{-1.1 \Delta y}
\end{aligned}
$$

The model was calibrated over the simulation time (Julian day: 222 - 284) by using a time step of 5 sec to allow for both model stability and model accuracy as discussed in Test 1.

The simulation was performed starting from the initial water surface elevation of the available data, $195.73 \mathrm{~m}$ at the dam. Figure 21 shows a comparison in water surface elevation between model predictions and field data. Such a comparison is necessary to check and verify the water balance accuracy. The model predictions showed good agreement with data. Error statistics based on field data taken daily showed that the root mean squared error (RMSE) was $0.079 \mathrm{~m}$ and the absolute mean error (AME) was $0.065 \mathrm{~m}$. The main target of this case study was to verify the ability of the model predictions to match the temperature profile field data over various times during the simulation period. The available data were at model location of $i=11$ and $j=5$ at Julian days (227.5, 232.5, 236.5, 239.5, 242.5, 248.5, 253.5, 257.5, 267.5, and 271.5). The water temperature calibration was performed by adjusting the temperature calibration parameters (Light extinction coefficient, evaporation coefficient, percent of light absorbed at the water surface, and wind-sheltering coefficient correcting for off-site wind data), described in details by Cole and Wells (2015). As a result, the model predictions of temperature in Lake Champlain model were primarily sensitive to the wind velocity on the lake. Figure 22 shows the model predictions of temperature compared to field data at various times. The comparison error statistics showed good agreement, reflecting the model's ability to predict temperatures successfully. Thus, the 3D model has been shown to predict water level and thermal structure of a stratified lake.

\section{Conclusions}

A 3D hydrodynamic and water quality model was developed by expanding the 2D fully implicit scheme of CE-QUAL-W2 in three dimensions. This model was novel in that it included simultaneous solution of hydrodynamics and water quality, solved the 3D equations such that the numerical solution of the free surface equation resulted in a tri-diagonal matrix solution, used selective withdrawal for reservoir outflows to avoid solving the full vertical momentum equation, and was built on the well-tested algorithms found in CE-QUAL-W2 model for water quality.

In order to validate the 3D numerical model, comparisons between the numerical solution and analytical solution were performed. These comparisons test the structure of the model and model assumptions. The model showed good agreement with the analytical solution for free surface seiching in a 
closed rectangular basin, free water surface response to wind-induced flow in a closed rectangular basin, and velocity profile response to the wind induced flow in a closed rectangular basin. For the seich induced flow, even though the model exhibited a stable solution, a lower time step showed a better match with the analytical solution. Hence, model stability does not guarantee model accuracy. Additionally, a field case study was performed on Lake Chaplain in Washington, USA. The case study exercised the model hydrodynamics linked with the heat balance equation. This comparison also showed that the model was able to reproduce field data successfully.

\section{Acknowledgments}

Many thanks to the Department of Civil and Environmental Engineering / Portland State University / Portland, OR USA, for the help in doing this research, funded by the Ministry of Higher Education and Scientific Research (MOHESR) - Iraq

\section{Funding}

This work was supported by the Ministry of Higher Education and Scientific Research (MOHESR) / University of Babylon - Iraq while Hussein Al-Zubaidi was a PhD student at Portland State University.

\section{Notation}

$a 1$ and $a 2$

$=$ randomly selected value of a variable

AME

$=$ absolute mean error

$b$

$=$ half length of the seiching basin

$\mathrm{B}$

$=$ width of the seiching basin

$c_{p}$

$=$ specific heat of water

$C_{Z}$

$=$ Chezy friction coefficient

$C_{D}$

$=$ drag coefficient

$D_{x}, D_{y}$, and $D_{z}$

$=$ diffusivities in the $x, y$, and $z$-direction, respectively

$f$

$=$ Coriolis parameter

$f_{*}$

$=$ reciprocal Coriolis parameter

$F_{x}$ and $F_{y}$

$=$ explicit operators

$g$

$g_{x}$

= gravitational acceleration

$g_{z}$

$=$ gravitational acceleration component in the $x$-direction

$h$

$=$ gravitational acceleration component in the $z$-direction

= bottom elevation 


$$
\begin{aligned}
& H \quad \text { = water depth of the grid cells or the seiching basin } \\
& H_{i, j, k} \quad \text { = water depth at the center of the grid cells } \\
& H_{r x} \quad \text { = water depth at the grid right face in the } x \text {-direction } \\
& H_{r y} \quad \text { = water depth at the grid right face in the } y \text {-direction } \\
& i, j \text {, and } k \\
& \text { imax, jmax, and kmax } \\
& k t \text { and } k b \\
& \mathrm{~K} \\
& \text { K } \\
& \text { l } \\
& \text { L } \\
& n \\
& p \\
& p_{a} \\
& \text { RMSE } \\
& R \\
& \mathrm{Ri} \\
& S_{\phi} \\
& t \\
& \text { T } \\
& \bar{u} \\
& u_{i} \\
& u^{\prime} \\
& u * \\
& U \\
& U_{s} \\
& \bar{v} \\
& \bar{w} \\
& W_{h} \\
& W_{x} \text { and } W_{y} \\
& W_{Z} \\
& W_{z 1} \\
& x, y \text {, and } z \\
& \text { = coordinate references for the finite differencing } \\
& =\text { maximum number of } i, j \text {, and } k \text {, respectively } \\
& \text { = top and bottom boundary cells, respectively } \\
& =\text { Von Karman constant } \\
& =\text { bottom cell } k \text { value } \\
& =\text { vertical mixing length scale } \\
& =\text { length of the seiching basin } \\
& =\text { time level } \\
& =\text { pressure } \\
& =\text { atmospheric pressure at the free surface elevation } \\
& =\text { root mean squared error } \\
& =\text { first stage numerical solution } \\
& =\text { Richardson number } \\
& =\text { source/sink term of the constituent } \phi \\
& =\text { time } \\
& \text { = water temperature (Celsius) } \\
& =\text { average velocity in the } x \text {-direction } \\
& =\text { velocity component in the } i \text {-direction } \\
& =\text { fluctuation of the velocity component } \\
& =\text { surface shear velocity } \\
& =W_{h}-U_{s} \\
& \text { = surface shear velocity. } \\
& =\text { average velocity in the } y \text {-direction } \\
& =\text { average velocity in the } z \text {-direction } \\
& =\text { wind velocity at height } h \\
& =\text { wind velocities in the } x \text { and } y \text {-direction, respectively } \\
& =\text { wind velocity at elevation } z \\
& =\text { wind velocity at elevation } z 1 \\
& \text { = Cartesian coordinate system axes }
\end{aligned}
$$




\begin{tabular}{|c|c|}
\hline$Z \circ$ & $=$ wind roughness height \\
\hline$z 1$ & $=$ elevation above the water surface \\
\hline$[\mathbf{A}]$ & $=$ diagonally predominant coefficients matrix \\
\hline$[B]$ & $=$ right hand side coefficients column matrix \\
\hline$[X]$ & $=$ unknown column matrix \\
\hline$\Delta t$ or $\mathrm{dt}$ & = time step \\
\hline$\Delta x, \Delta y$, and $\Delta z$ & = grid resolution \\
\hline$\Omega$ & $=$ earth rotation rate \\
\hline$\varphi$ & = latitude \\
\hline$\alpha$ & = waterbody slope \\
\hline$\gamma_{i, j, k}$ and $\alpha_{i, j, k}$ & $=$ operators for Thomas algorithm \\
\hline$v_{t j}$ & $=$ turbulent kinematic viscosity in the $j$-direction \\
\hline$v_{t}$ & $=$ constant vertical eddy viscosity \\
\hline$v_{h}$ & $=$ horizontal turbulent kinematic viscosity \\
\hline$v_{v}$ & $=$ vertical turbulent kinematic viscosity \\
\hline$v_{m}$ & $=$ minimum turbulent kinematic viscosity \\
\hline$\eta$ & = free surface elevation \\
\hline$\tau^{x x}, \tau^{x y}, \tau^{x z}, \tau^{y x}, \tau^{y y}, \tau^{y z}, \tau^{z x}, \tau^{z y}$, and $\tau^{z z}$ & $=$ viscous shear stresses \\
\hline$\tau_{x x}, \tau_{x y}, \tau_{x z}, \tau_{y x}, \tau_{y y}, \tau_{y z}, \tau_{z x}, \tau_{z y}$, and $\tau_{z z}$ & $=$ turbulent shear stresses or Reynolds stresses. \\
\hline$\tau_{s}$ and $\tau_{b}$ & = surface and bottom shear stresses, respectively \\
\hline$\tau_{s x}$ and $\tau_{s y}$ & $=$ surface shear stresses in the $x$ and $y$-direction, respectively \\
\hline$\tau_{b x}$ and $\tau_{b y}$ & $=$ bottom shear stresses in the $x$ and $y$-direction, respectively \\
\hline$\rho$ and $\rho_{w}$ & $=$ water density \\
\hline$\rho_{a}$ & = air density \\
\hline$\theta_{1}$ & $=$ angle that wind makes with the northern direction \\
\hline$\theta_{2}$ & $=$ angle that the $x$-direction makes with the northern direction \\
\hline$\phi$ & $=$ water quality constituent concentration \\
\hline
\end{tabular}

\section{References}

Ahsan, A. K. M., \& Blumberg, A. F. (1999). Three-dimensional hydrothermal model of Onondaga Lake, New York. Journal of Hydraulic Engineering, 125(9), 912-923.

Annear, R. L., Berger, C. J., \& Wells, S. A. (2008). Lake Chaplain model: Model development, calibration, and management scenarios (Report No. EWR-01-08). Portland, OR: Water Quality 
Research Group, Department of Civil and Environmental Engineering, Portland State University.

Barron, C. N., Kara, A. B., Martin, P. J., Rhodes, R. C., \& Smedstad, L. F. (2006). Formulation, implementation and examination of vertical coordinate choices in the global Navy Coastal Ocean Model (NCOM). Ocean Modelling, 11, 347-375.

Blumberg, A. F., \& Mellor, G. L. (1980). A coastal ocean numerical model. Proc., Int. Symp. on Math. Modelling of Estuarine Phys., J. Sundermann and K. P. Holz, Eds., Springer, Berlin, 202-19.

Blumberg, A. F., \& Mellor, G. L. (1987). A description of a three-dimensional coastal ocean circulation model. Three-Dimensional Coastal Ocean Models. N. S. Heaps, Ed., American Geophysical Union, Washington, D.C., 1-16.

Bryan, K. (1969). A numerical method for the study of the circulation of the world ocean. Journal of Computational Physics, 4(3), 347-376.

Casulli, V., \& Cheng, R. T. (1992). Semi-implicit finite difference methods for three-dimensional shallow water flow. International Journal for Numerical Methods in Fluids, 15(6), 629-648.

Casulli, V., \& Walters, R. (2000). An unstructured grid, three-dimensional model based on the shallow water equations. International Journal for Numerical Methods in Fluids, 32, 331-348.

Chen, C., Beardsley, R. C., Cowles, G., Qi, J., Lai, Z., Gao, G., ... Lin, H. (2011). An unstructured-grid, finite-volume community ocean model FVCOM user manual, 3rd edition (Report No. 11-1101). Cambridge, Massachusetts: Massachusetts Institute of Technology,

Chen, C., Liu, H., \& Beardsley, R. C. (2003). An unstructured grid, finite-volume, three-dimensional, primitive equations ocean model: Application to coastal ocean and estuaries. Journal of Atmpspheric and Oceanic Technology, 20, 159-186.

Cole, T., \& Buchak, E. (1995). CE-QUAL-W2: A two-dimensional, laterally averaged, hydrodynamic and water quality model, version 2.0 (Instruction Rep. EL-95-1). Washington, DC: U.S. Army Corps of Engineers.

Cole, T., \& Wells, S. A. (2015). CE-QUAL-W2: A two-dimensional, laterally averaged, hydrodynamic and water quality model (version 3.72). Portland, OR: Department of Civil and Environmental Engineering, Portland State University.

Csanady, G. T. (2013). Circulation in the coastal ocean. Netherlands: Springer.

Cushman-Roisin, B., \& Beckers, J. M. (2007). Introduction to geophysical fluid dynamics: Physical and numerical aspects. Academic Press.

Dronkers, J. J. (1964). Tidal computations in rivers and coastal seas. Amsterdam: North Holland Publishing Co.

Edinger, J. E. (2001). Waterbody hydrodynamic and water quality modeling: An introductory workbook and CD-ROM on three-dimensional waterbody modeling. Virginia, USA: ASCE. 
Eliason, D. E., \& Bourgeois, A. J. (1997). Validation of numerical shallow water models for stratified seiches. International Journal for Numerical Methods in Fluids, 24(8), 771-786.

EPA. (1971). Effect of geographical location on cooling pond requirements and performance (Report No. 16130 FDQ in water pollution control research series). Washington, DC: Water Quality Office, Environmental Protection Agency.

Hamrick, J. M. (1992). A three-dimensional environmental fluid dynamics computer code: Theoretical and computational aspects (Special Report 317 in applied marine science and ocean engineering). Virginia: Virginia Institute of Marine Science, School of Marine Science, College of William and Mary.

Hansen, N. E. (1975). Entrainment in two-layered flows, Institute of Hydrodynamics and Hydraulic Engineering. Technical Institute of Denmark, Series Paper No. 7.

Hodges, B., \& Dallimore, C. (2006). Estuary, lake and coastal ocean model: ELCOM (v2.2 Science Manual). Australia: Center for Water Research, University of Western.

Imberger, J. and Fischer, H. B. (1970). Selective withdrawal from a stratified reservoir (Report No. 15040 EJZ). Washington, DC: Environmental Protection Agency.

Kowalik, Z., \& Murty, T. S. (1993). Numerical modeling of ocean dynamics. Singapore: World Scientific.

Leendertse, J. J., \& Liu, S. K. (1975). A three-dimensional model for estuaries and coastal seas: Vol. II, aspects of computation (R-1764-OWRT). Santa Monica, CA: Retrieved from Rand Corporation website: https://www.rand.org/pubs/reports/R1764.html

Leonard, B. P. (1979). A stable and accurate convective modelling procedure based on quadratic upstream interpolation. Computer Methods in Applied Mechanics and Engineering, 19, 59-98.

Leonard, B. P. (1991). The ULTIMATE conservative difference scheme applied to unsteady onedimensional advection. Computer Methods in Applied Mechanics and Engineering, 88, 17-74.

Martin, P. J., Barron, C. N., Smedstad, L. F., Campbell, A. J., Rhodes, R. C., Rowley, C., ... Carroll, S. N. (2009). User's manual for the navy coastal ocean model ( NCOM ) version 4.0 (Report No. NRL/MR/7320--08-9151). MS, USA: Naval Research Laboratory, Oceanography division, Stennis Space Center.

Mellor, G. L. (2002). Users guide for a three-dimensional, primitive equation, numerical ocean model (October 2002 version). Princeton, NJ: Program in Atmospheric and Oceanic Sciences, Princeton University.

Neumann, L. E., Simunek, J., \& Cook, F. J. (2011). Implementation of quadratic upstream interpolation schemes for solute transport into HYDRUS-1D. Environmental Modelling \& Software, 26, 12981308.

Okubo, A. (1971). Oceanic diffusion diagrams. Deep-Sea Research, 18(8), 789-802. 
Patankar, S. V. (1980). Numerical heat transfer and fluid flow. New York, USA: Hemisphere Publishing Corporation.

Ryan, P. J., \& Harleman, D. R. F. (1973). An analytical and experimental study of transient cooling pond behavior (Report No. 161). Cambridge, MA: Ralph M. Parsons Laboratory, Department of Civil Engineering, Massachusetts Institute of Technology.

Sheng, Y., \& Butler, H. (1982). Modeling coastal currents and sediment transport. Proceedings of the 18th international conference on coastal engineering (pp.1127-1148). Cape Town, South Africa: ASCE.

Smith, P. E. (2006). A semi-implicit, three-dimensional model for estuarine circulation (Open-File Report 2006-1004). Sacramento, CA: U.S. Geological Survey.

Tetra Tech, Inc. (2002). Draft user's manual for environmental fluid dynamic code Hydro Version (EFDC-Hydro) (Release 1.00). Fairfax, Virginia: Tetra Tech, Inc.

Vreugdenhil, C. B. (1989). Computational hydraulics: An introduction. Springer-Verlag Berlin Heidelberg.

Wang, H., \& Falconer, R. A. (1998). Numerical modeling of flow in chlorine disinfection tanks. Journal of Hydraulic Engineering, 124(9), 918-931.

Wang, S. Y., Roache, P. J., Schmalz, R. A., Jia, Y., \& Smith, P. E. (2009). Verification and validation of $3 D$ free-surface flow models. United States of America: ASCE.

Wells, S. A. (2002). Basis for the CE-QUAL-W2 version 3 river basin hydrodynamic and water quality model. Proceedings of the 2nd Federal Interagency Hydrologic Modeling Conference. Las Vegas, NV.

Wu, J. (1969). Wind stress and surface roughness at air-sea interface. Journal of Geophysical Research, 74(2), 444-455. 


\section{List of figures}

Figure 1 Positive direction coordinate system

Figure 2 Free surface integration limits

Figure 3 Variables distribution in a cell

Figure 4 Seiching basin for the test 1

Figure 5 Comparison in the water level $(\eta)$ between the model results and the analytical solution near the left and right boundary ( $i=3$ and $19, j=3$, and $k=k t=3$ ), $\Delta t=5 \mathrm{sec}$

Figure 6 Comparison in the longitudinal velocity $(u)$ between the model results and the analytical solution near the right boundary $(i=19, j=3$, and $k=k t=3), \Delta t=5 \mathrm{sec}$

Figure 7 Comparison in the longitudinal velocity $(u)$ between the model results and the analytical solution near the left boundary $(i=3, j=3$, and $k=k t=3), \Delta t=5 \mathrm{sec}$

Figure 8 Dumping effect on the computed water level wave using different time steps for the seiching basin near the right boundary ( $i=19, j=3$, and $k=k t=3$ )

Figure 9 Comparison in water level wave using two spatial resolutions at the same time step

Figure 10 Seiching basin for the test 2

Figure 11 The computed water level under the wind effect in a closed rectangular basin using different time steps near the left boundary ( $i=3, j=3$, and $k=k t=3)$

Figure 12 The computed water level under the wind effect in a closed rectangular basin using different time steps near the left and right boundaries ( $i=3$ and $i=19, j=3$, and $k=k t=3$ )

Figure 13 Seiching basin for the test 3

Figure 14 The computed and analytical velocity profile under the effect of wind induced flow in the middle of the seiching basin at time $=1000 \mathrm{sec}$

Figure 15 Lake Chaplain watershed

Figure 16 Lake Chaplain model computational grid and metric elevations

Figure 17 Lake Chaplain inflow time series

Figure 18 Lake Chaplain outflow time series to the paper mill

Figure 19 Lake Chaplain outflow time series to the water treatment plant

Figure 20 Lake Chaplain inflow time series of temperature

Figure 21 Model predictions of water surface elevation compared to field data

Figure 22 Model predictions of temperature compared to field data 


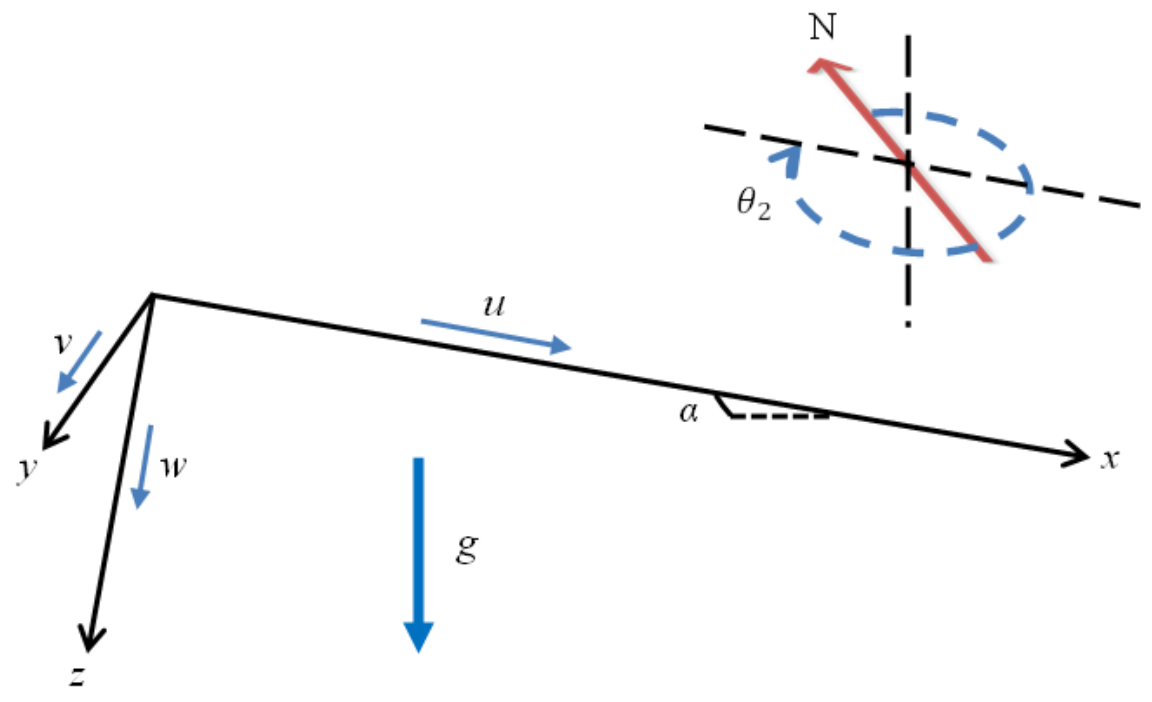

Figure 1 Positive direction coordinate system

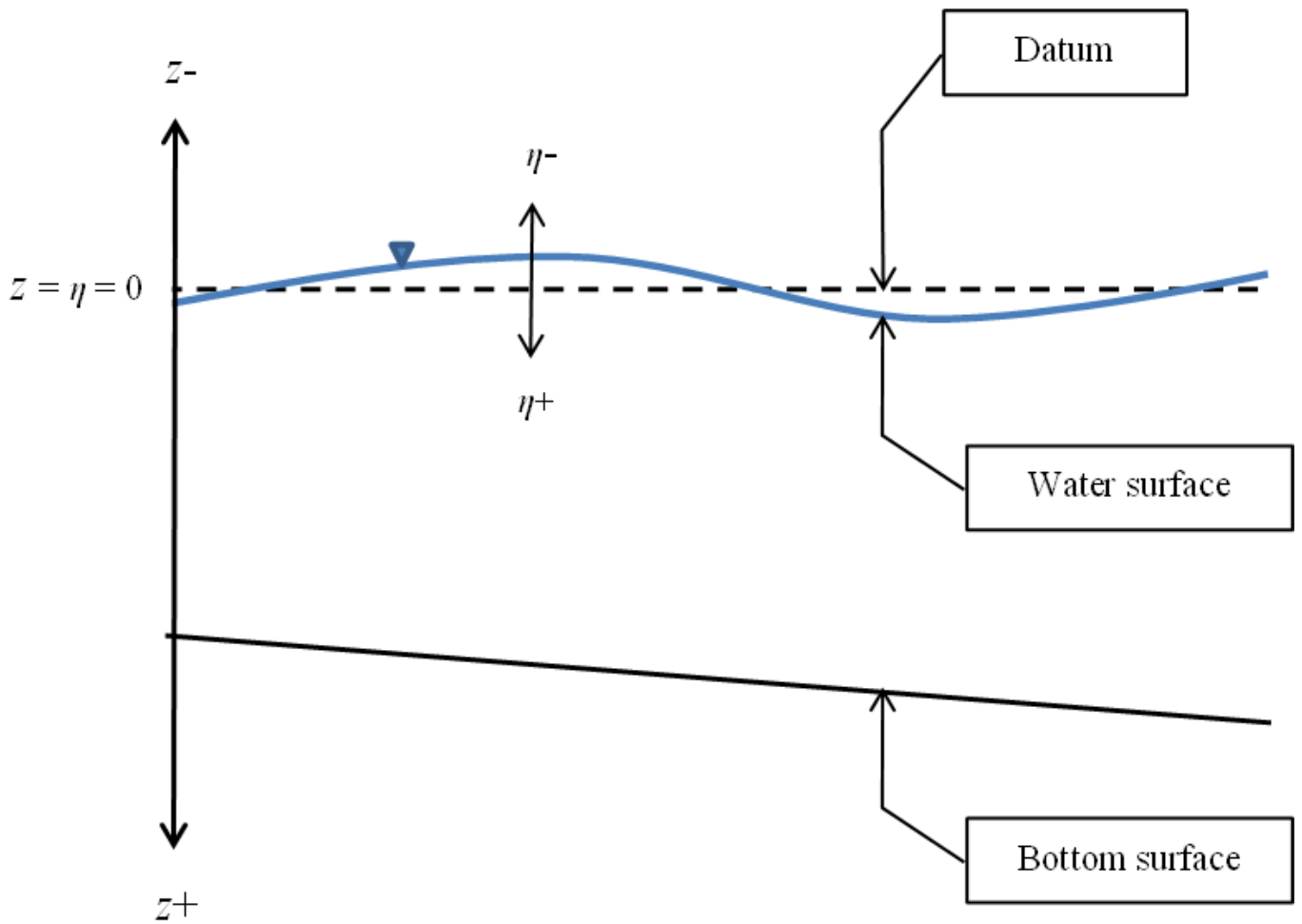

Figure 2 Free surface integration limits 

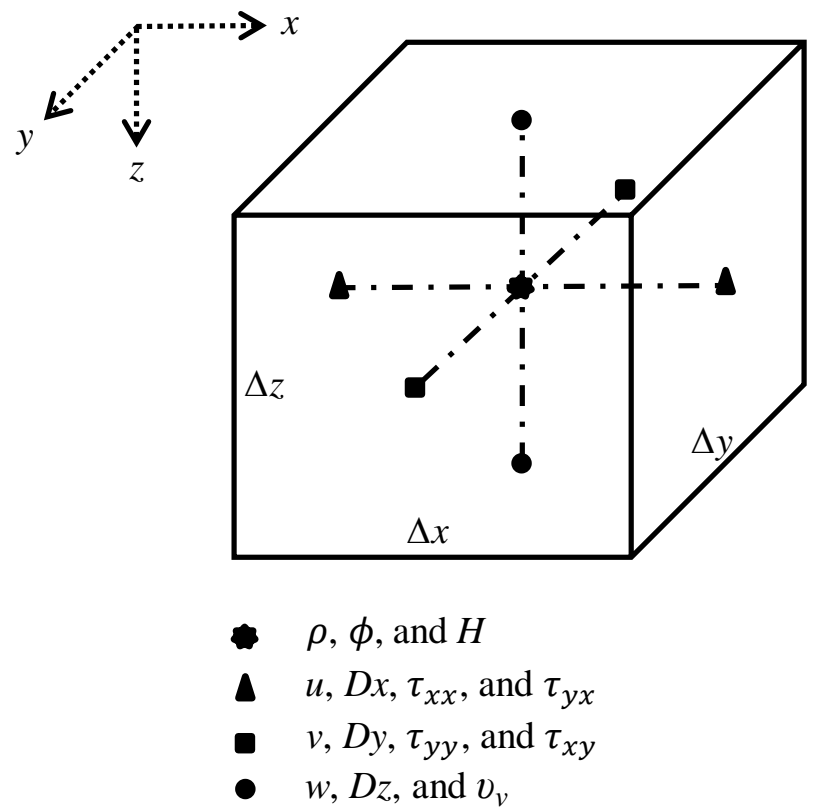

Figure 3 Variables distribution in a cell

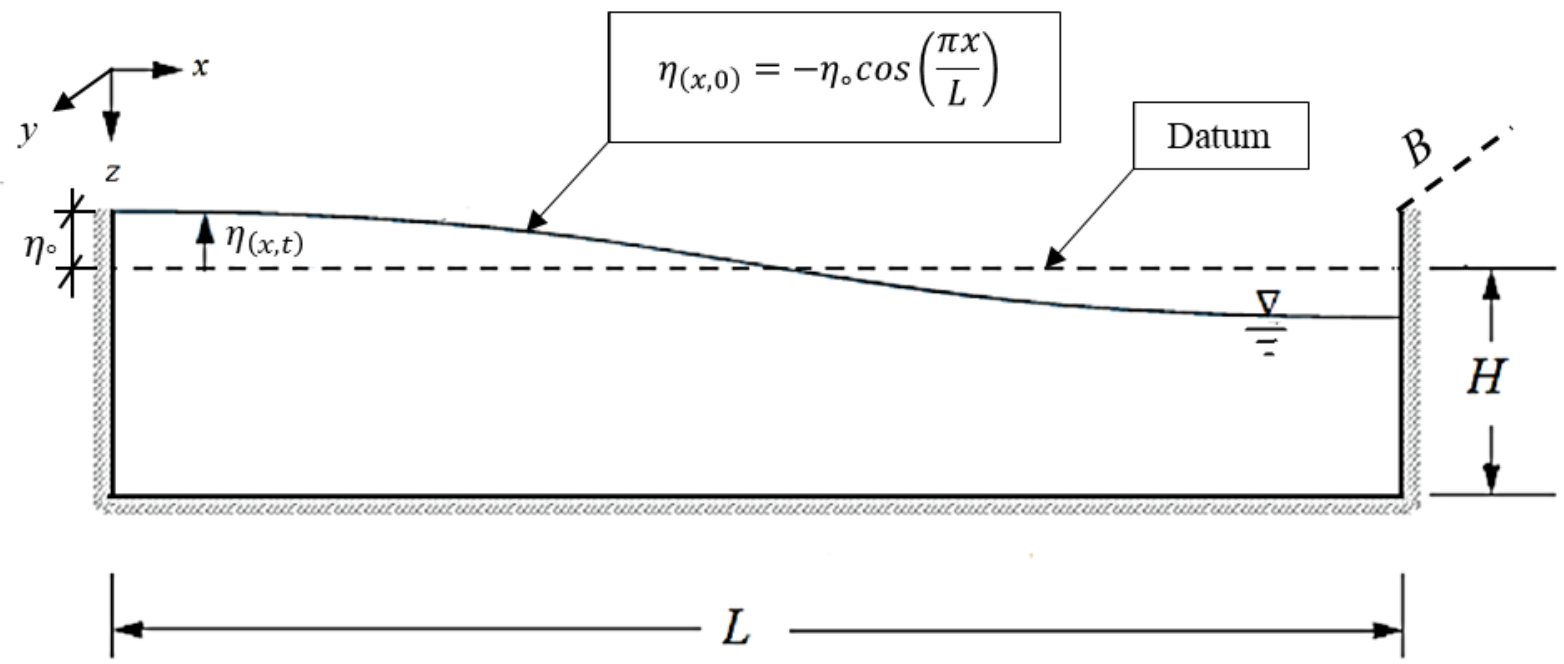

Figure 4 Seiching basin for the test 1 


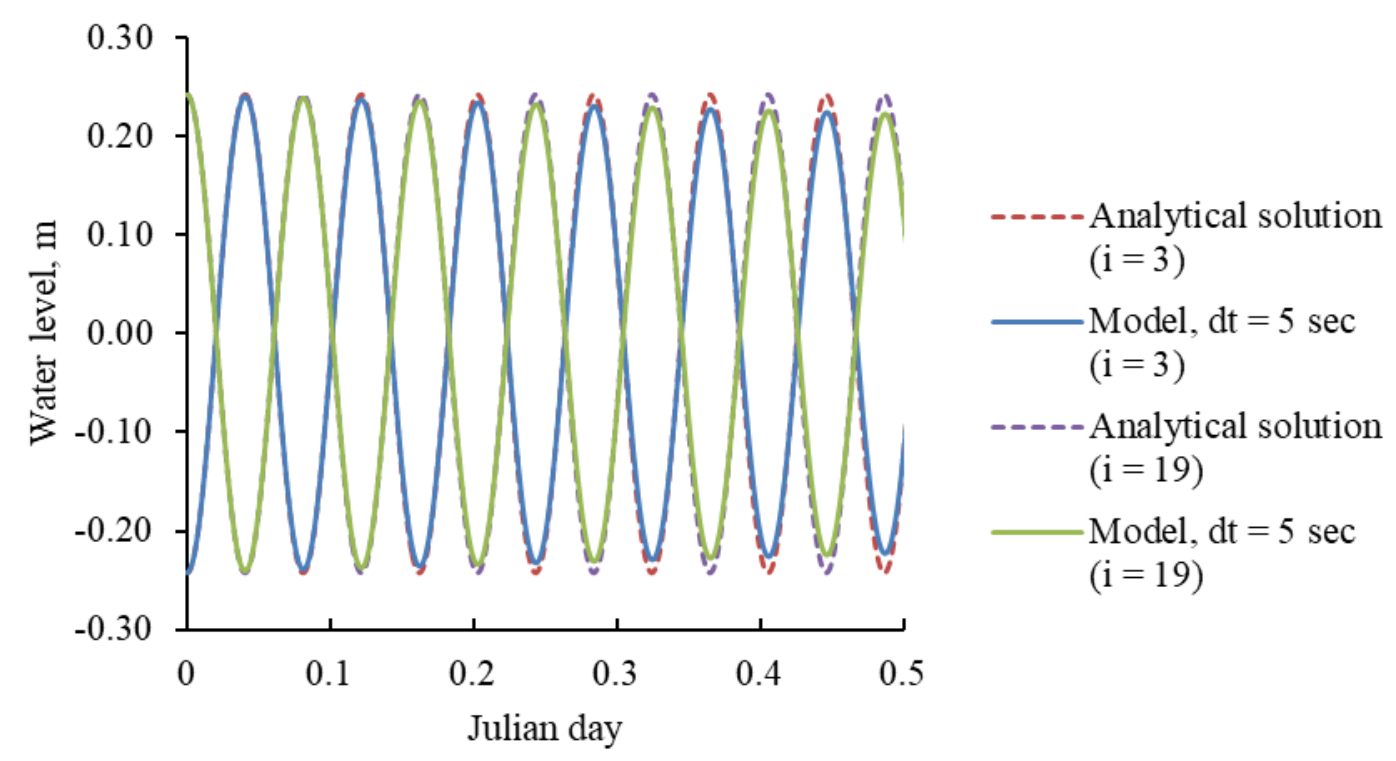

Figure 5 Comparison in the water level $(\eta)$ between the model results and the analytical solution near the left and right boundary ( $i=3$ and $19, j=3$, and $k=k t=3$ ), $\Delta t=5 \mathrm{sec}$

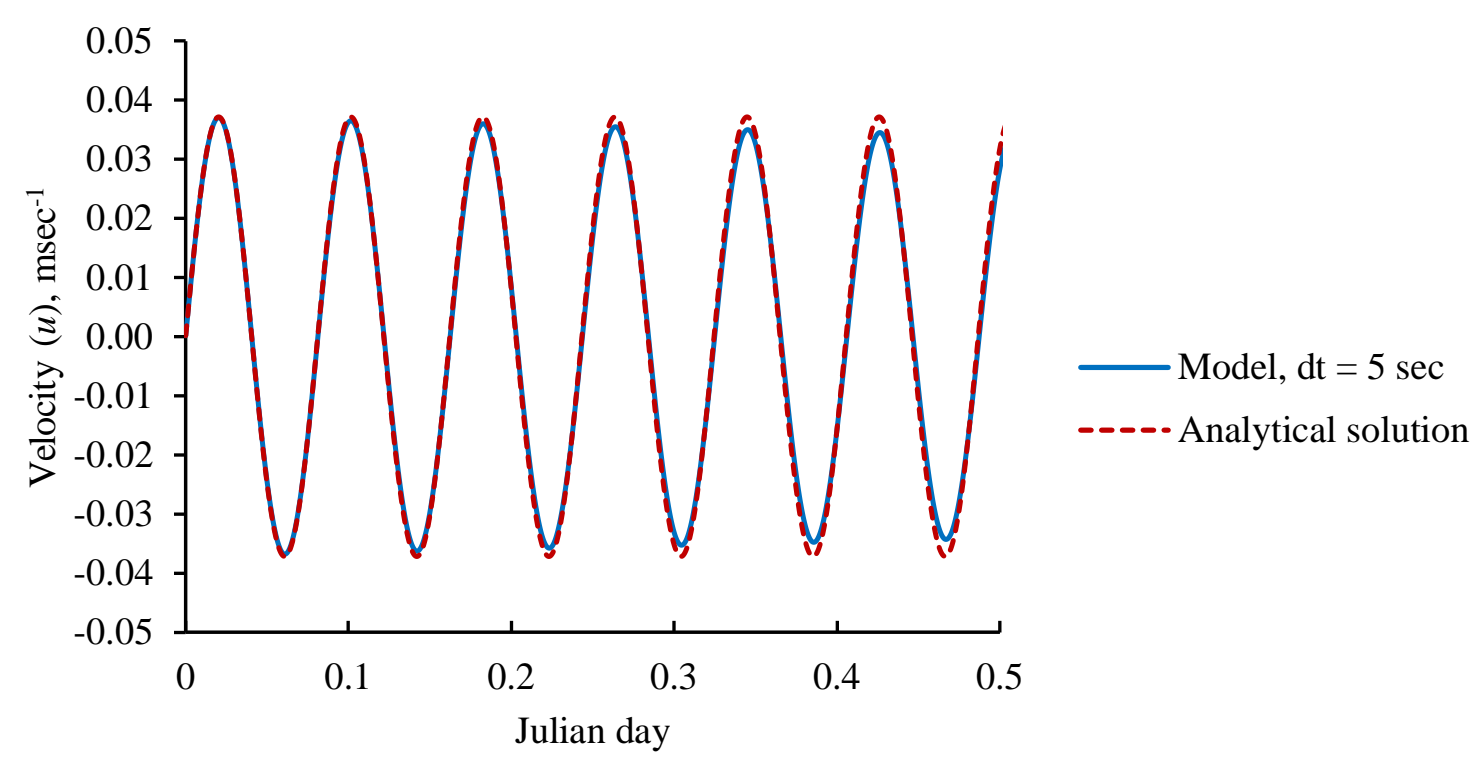

Figure 6 Comparison in the longitudinal velocity $(u)$ between the model results and the analytical solution near the right boundary ( $i=19, j=3$, and $k=k t=3$ ), $\Delta t=5 \mathrm{sec}$ 


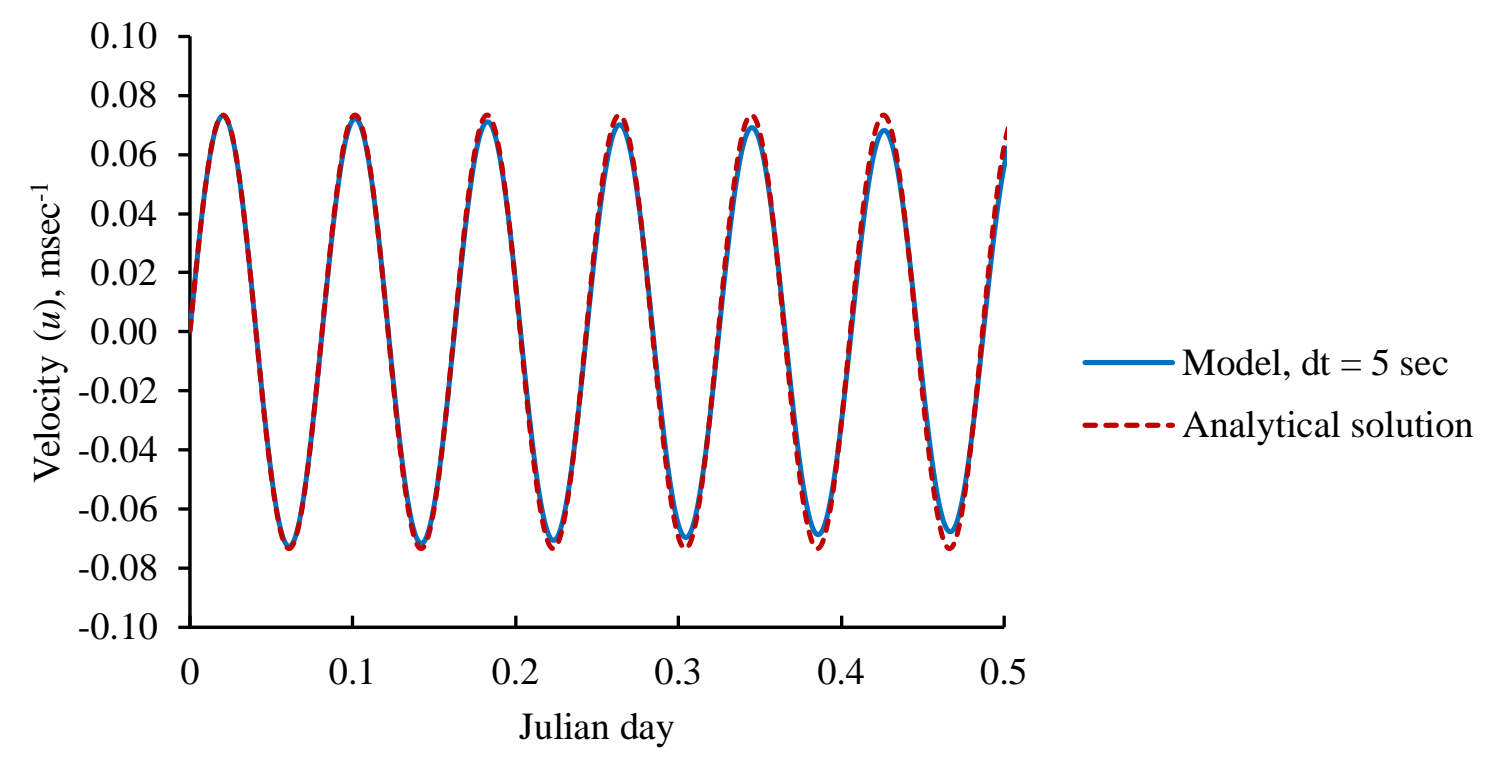

Figure 7 Comparison in the longitudinal velocity $(u)$ between the model results and the analytical solution near the left boundary $(i=3, j=3$, and $k=k t=3), \Delta t=5 \mathrm{sec}$

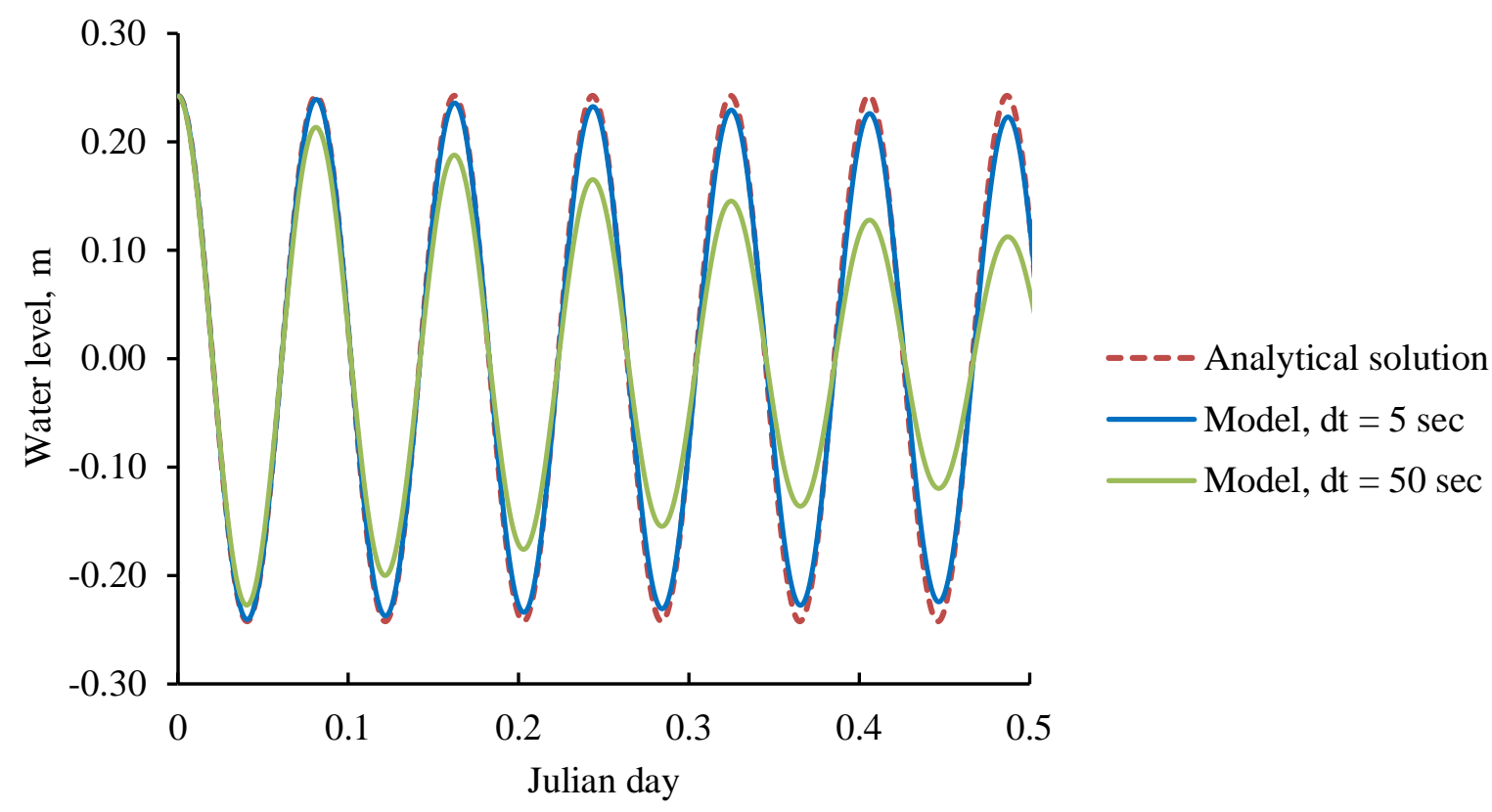

Figure 8 Dumping effect on the computed water level wave using different time steps for the seiching basin near the right boundary $(i=19, j=3$, and $k=k t=3)$ 


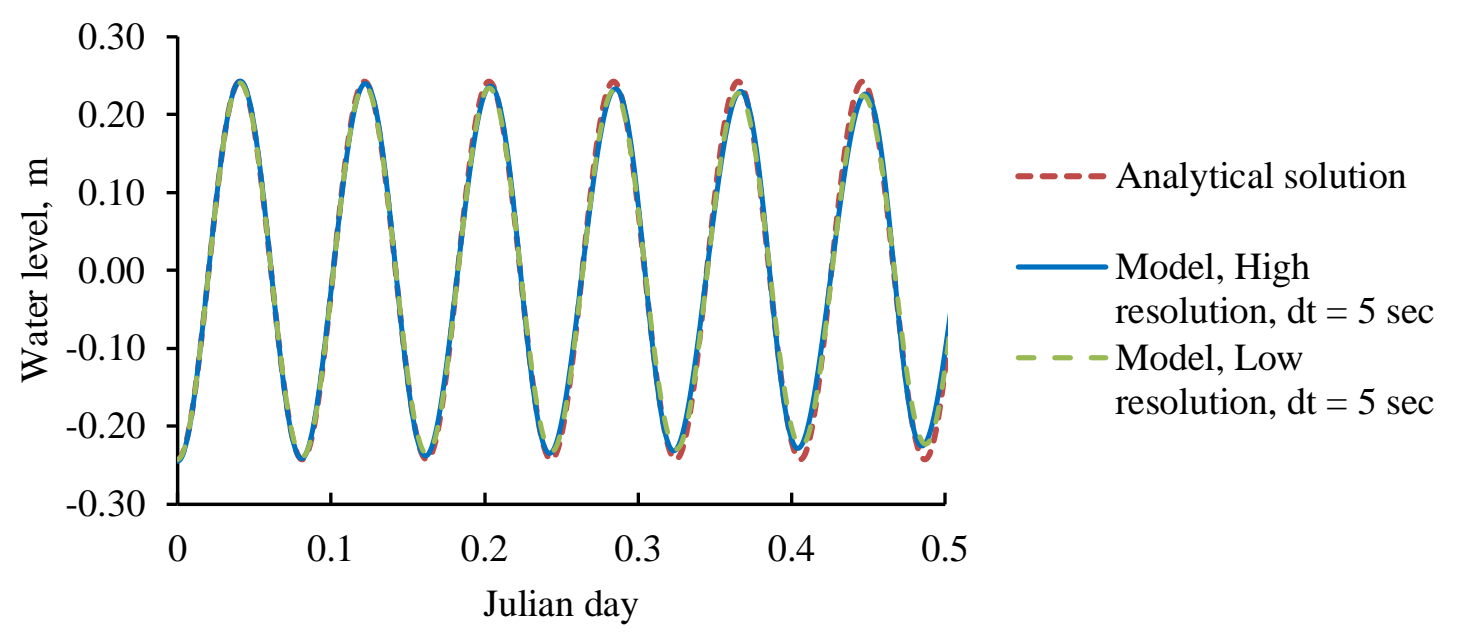

Figure 9 Comparison in water level wave using two spatial resolutions at the same time step

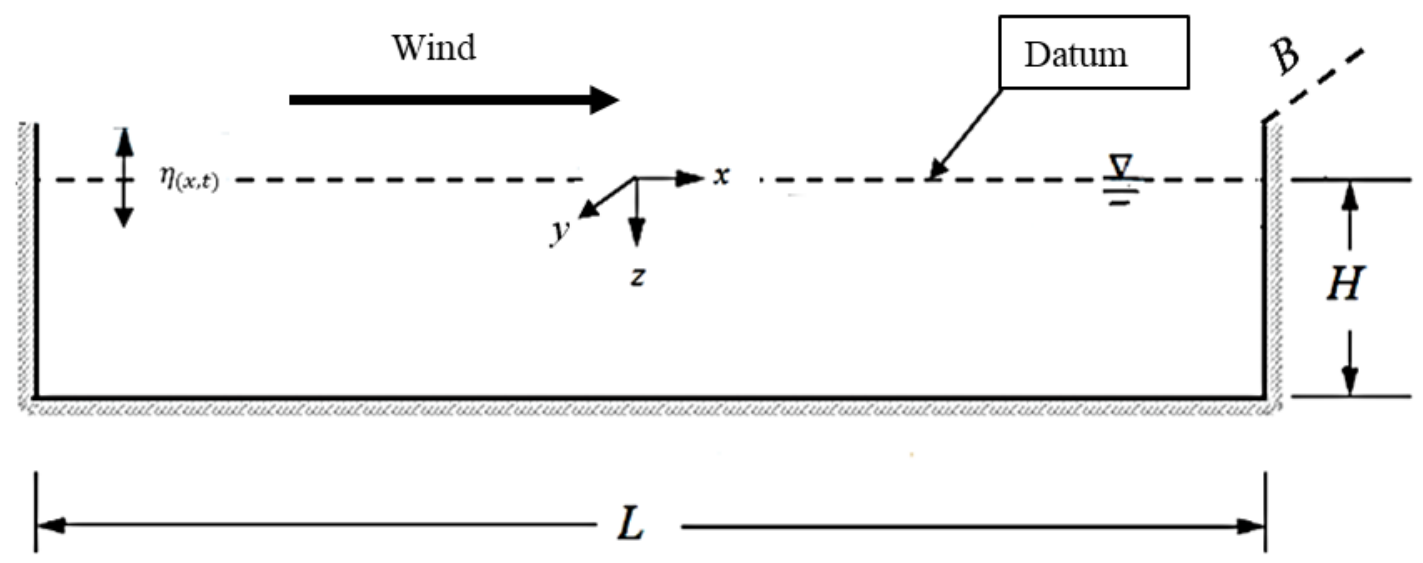

Figure 10 Seiching basin for the test 2 


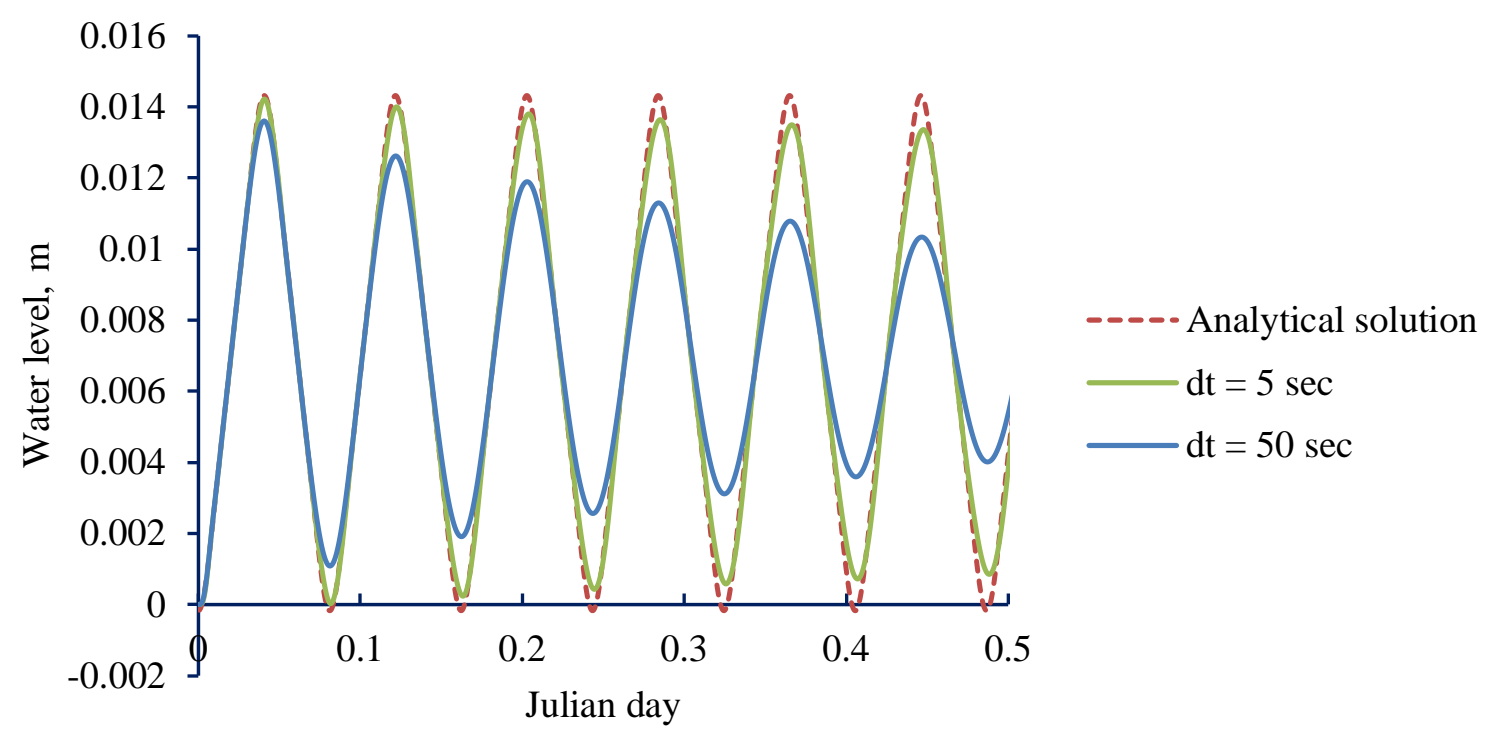

Figure 11 The computed water level under the wind effect in a closed rectangular basin using different time steps near the left boundary $(i=3, j=3$, and $k=k t=3)$

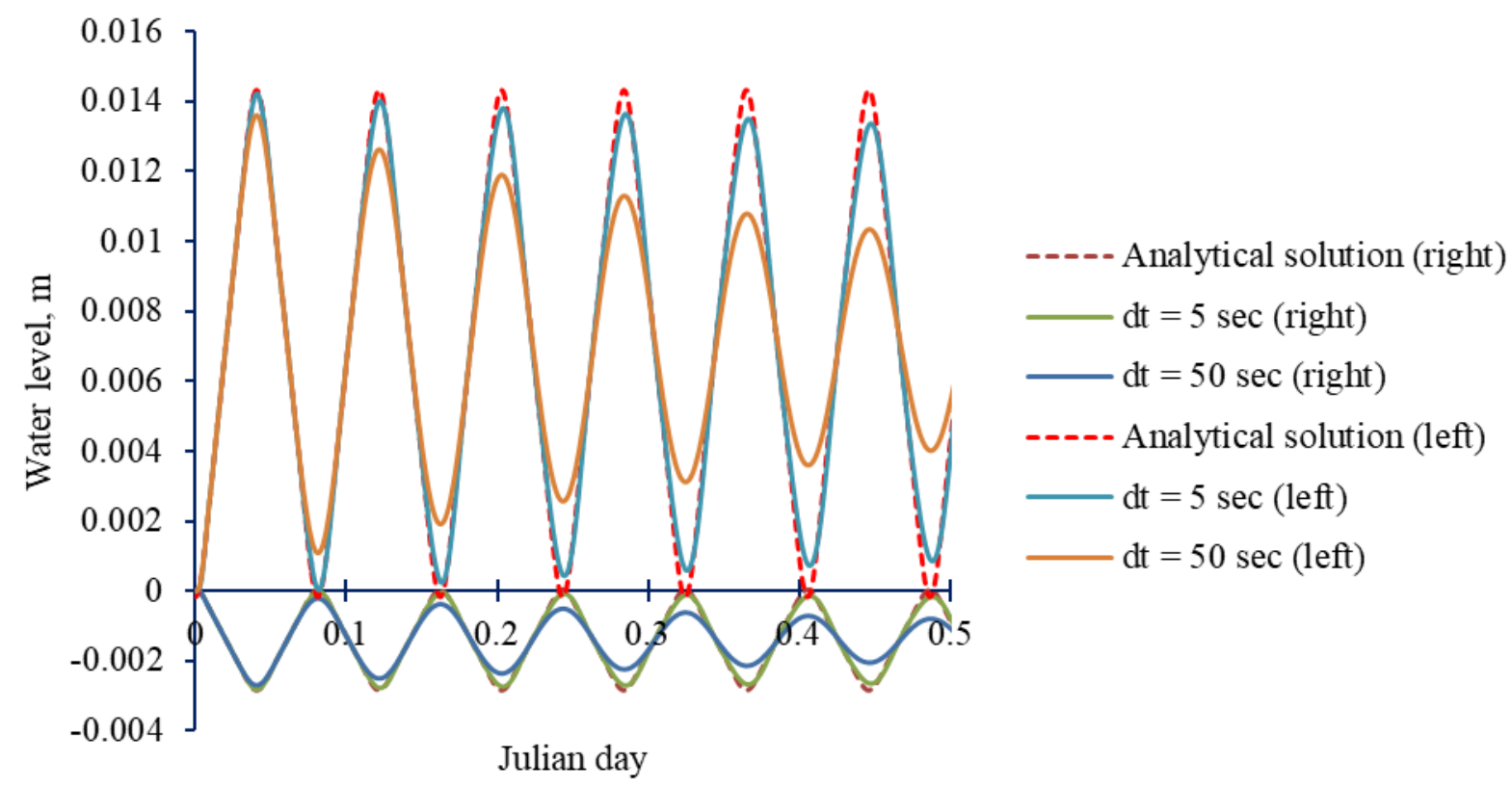

Figure 12 The computed water level under the wind effect in a closed rectangular basin using different time steps near the left and right boundaries ( $i=3$ and $i=19, j=3$, and $k=k t=3$ ) 


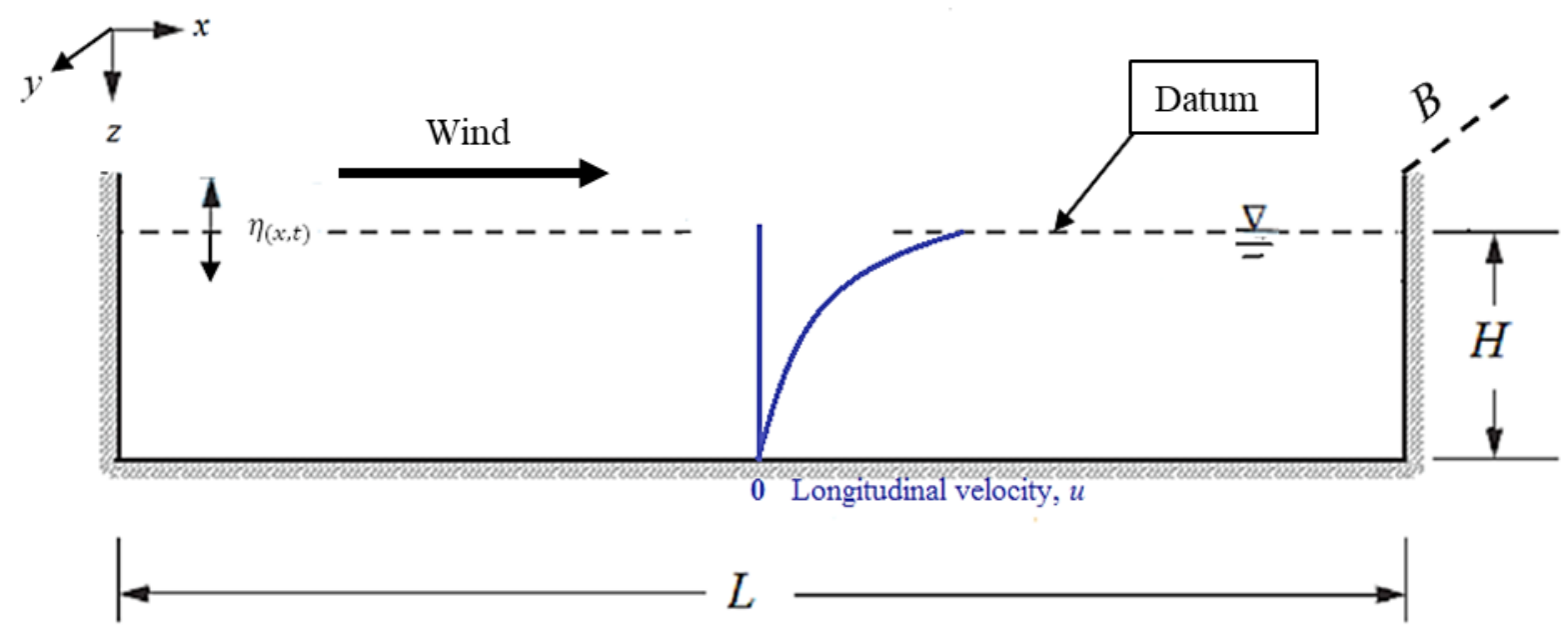

Figure 13 Seiching basin for the test 3

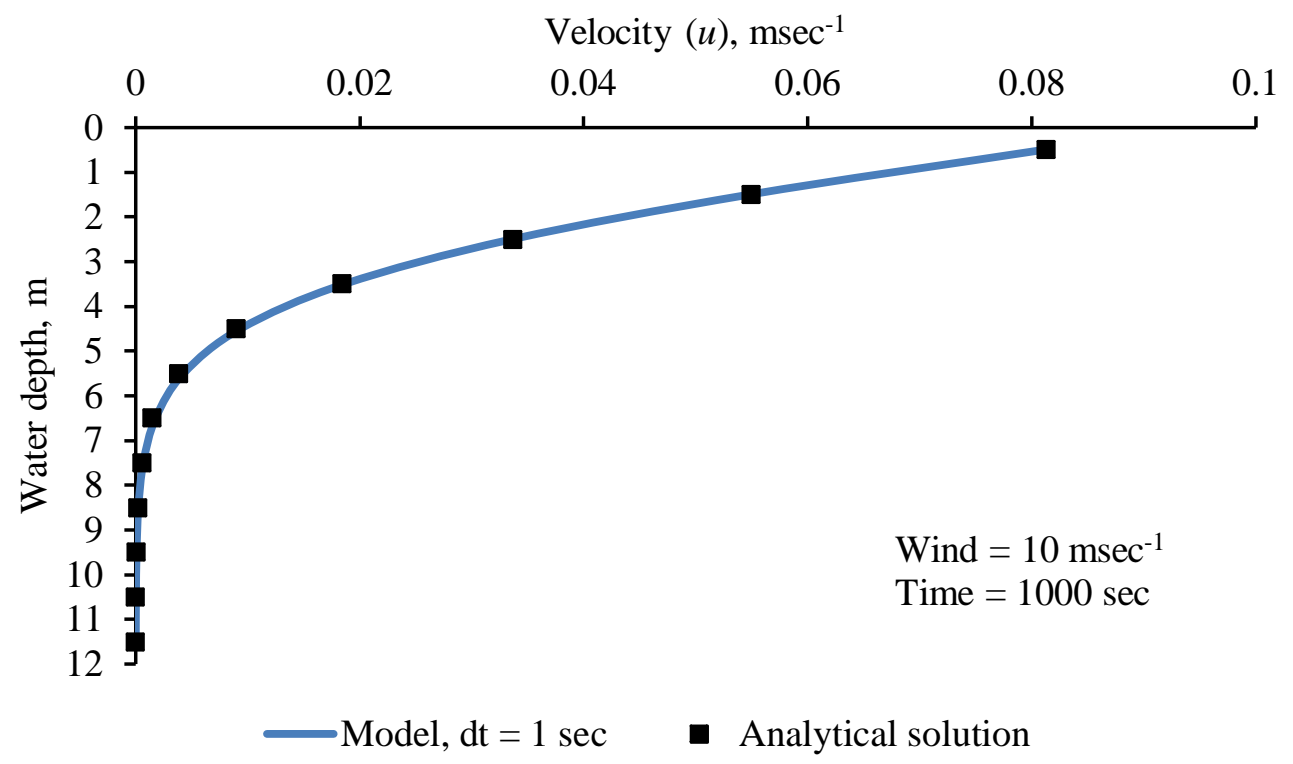

Figure 14 The computed and analytical velocity profile under the effect of wind induced flow in the middle of the seiching basin at time $=1000 \mathrm{sec}$ 


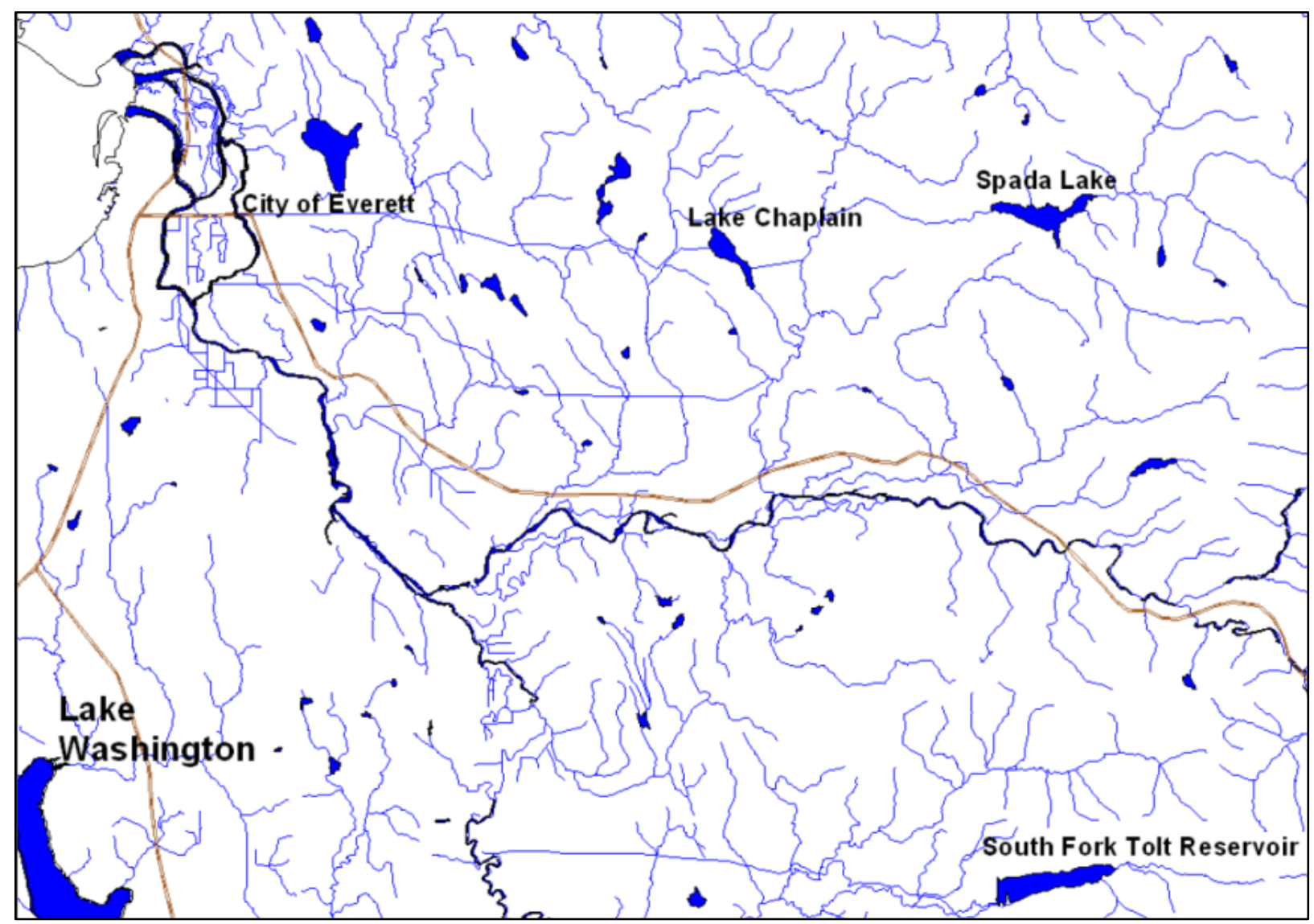

Figure 15 Lake Chaplain watershed 


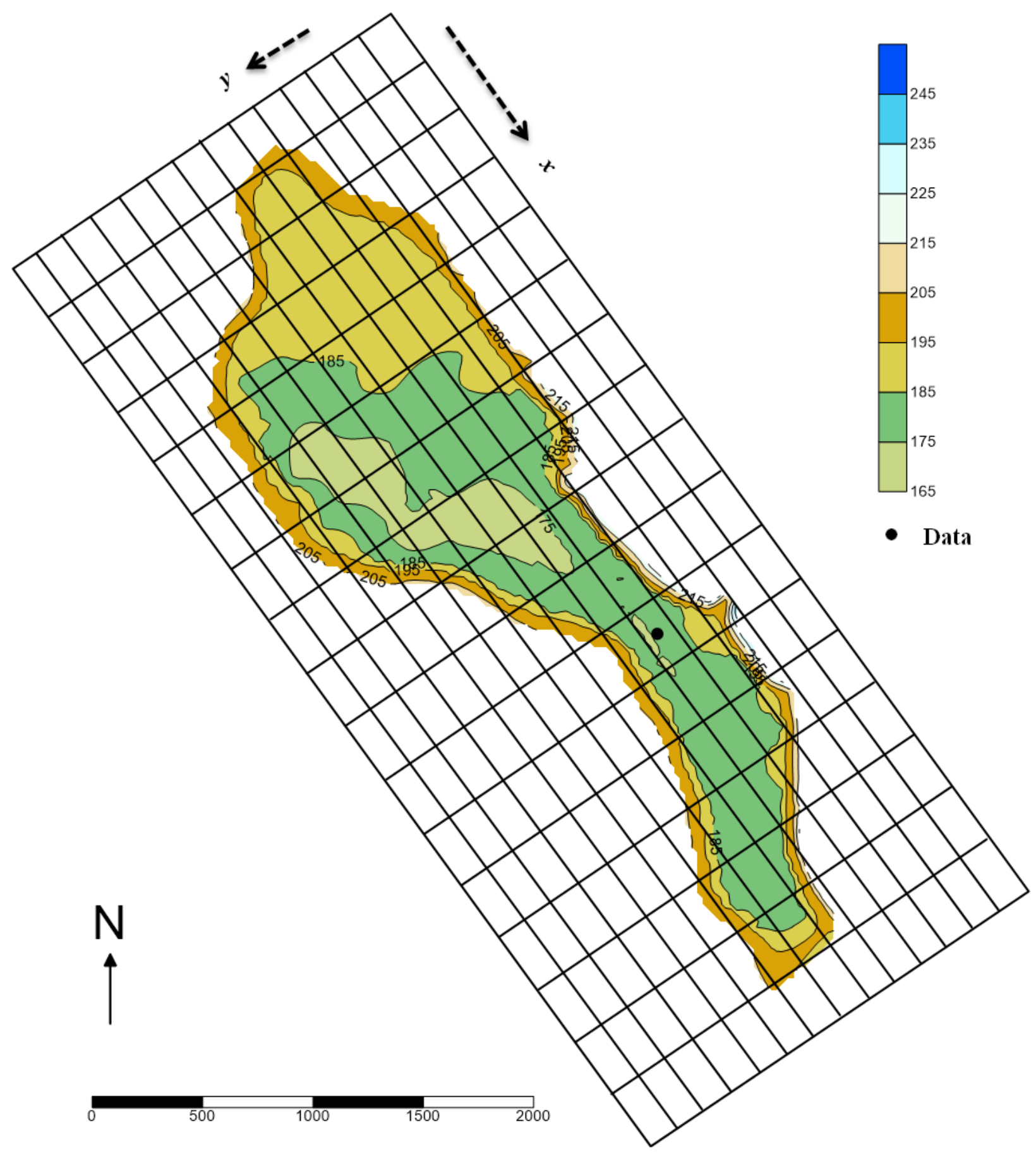

Figure 16 Lake Chaplain model computational grid and metric elevations 


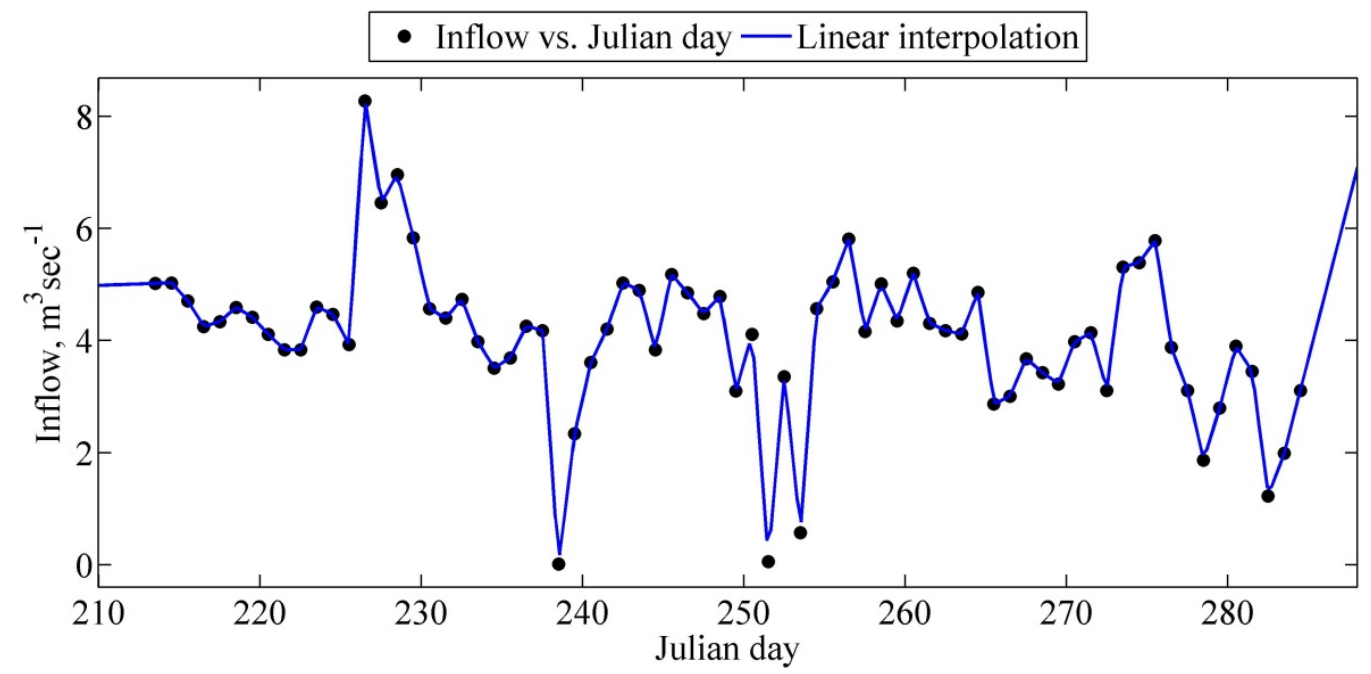

Figure 17 Lake Chaplain inflow time series

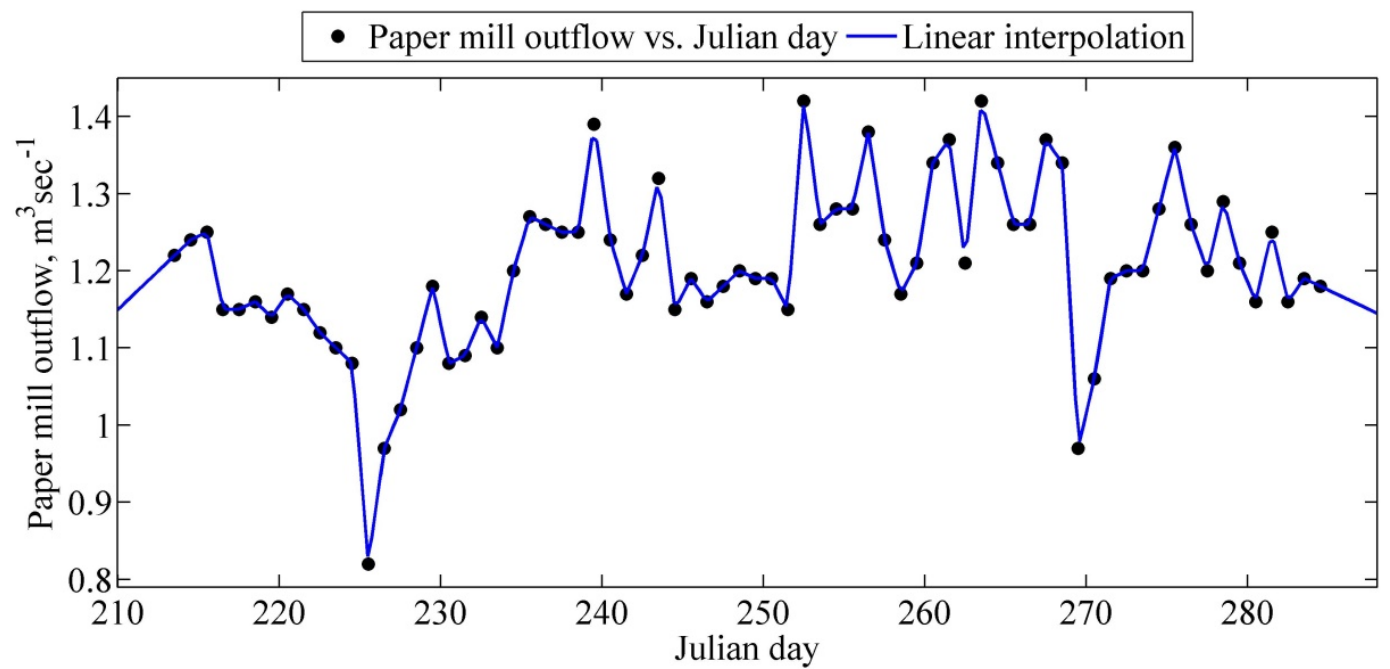

Figure 18 Lake Chaplain outflow time series to the paper mill 


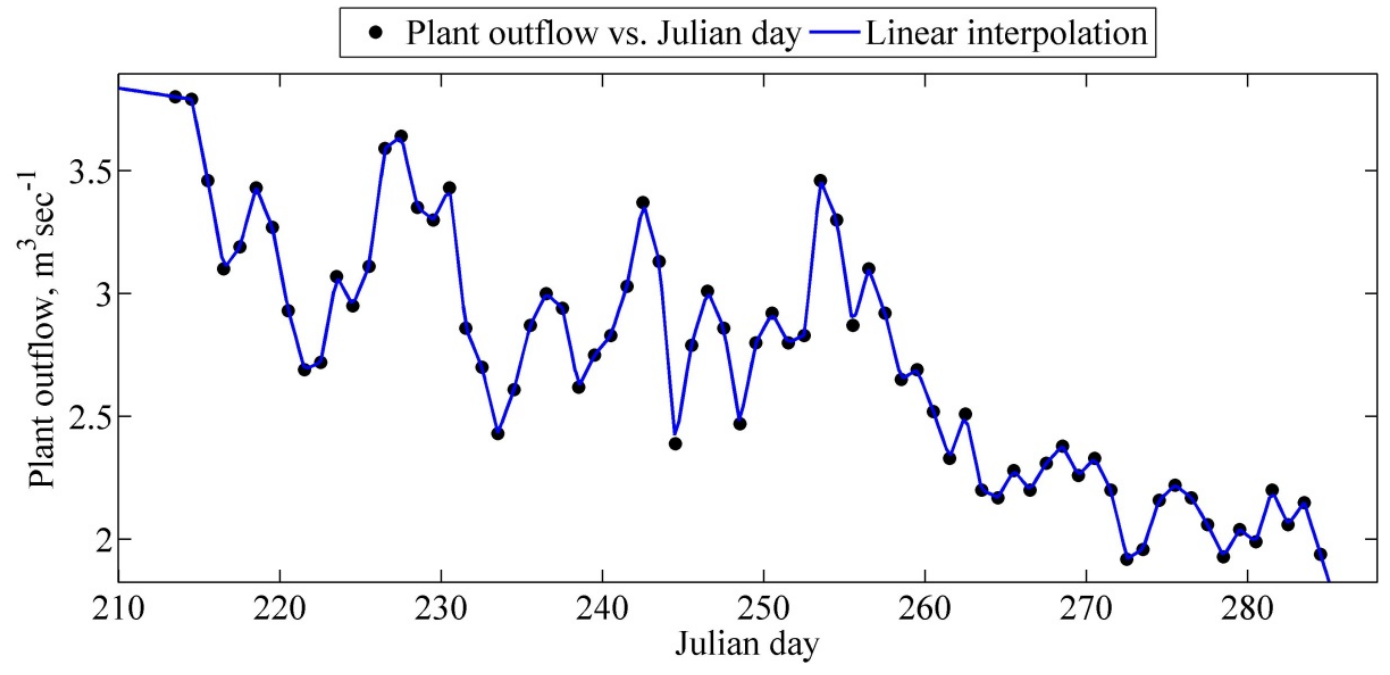

Figure 19 Lake Chaplain outflow time series to the water treatment plant

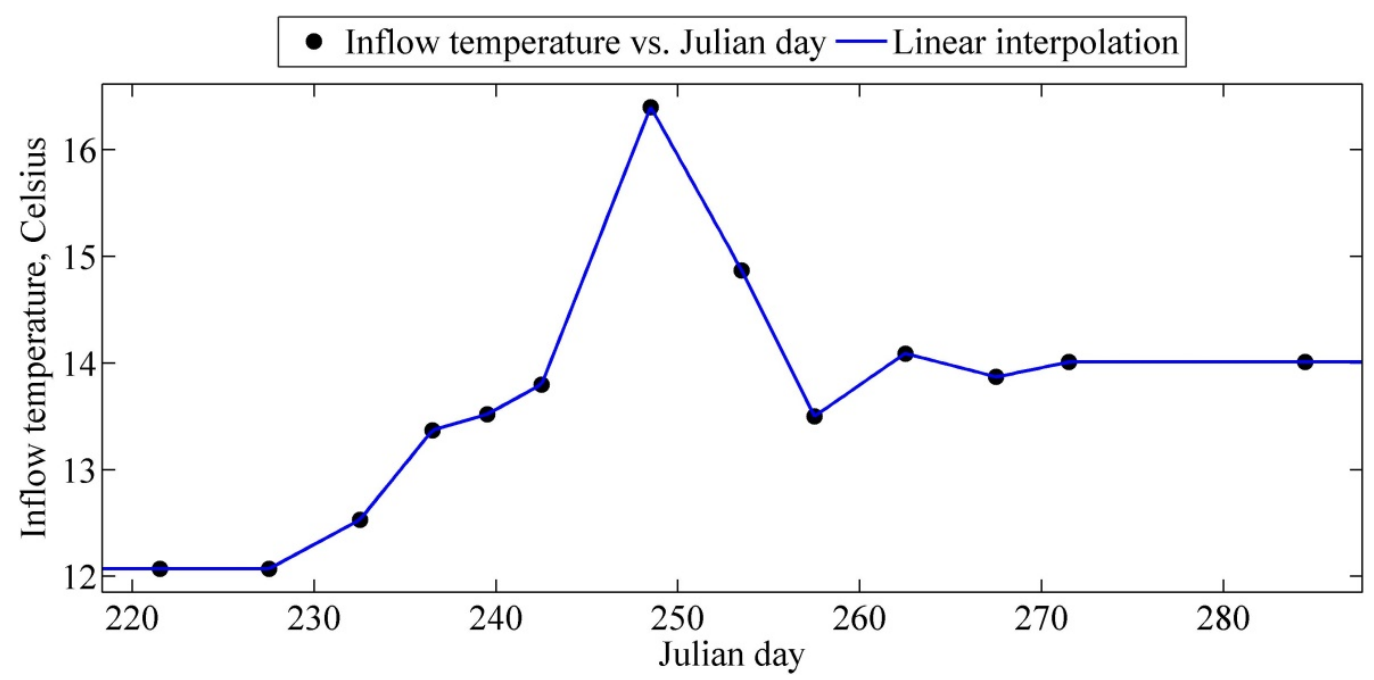

Figure 20 Lake Chaplain inflow time series of temperature 


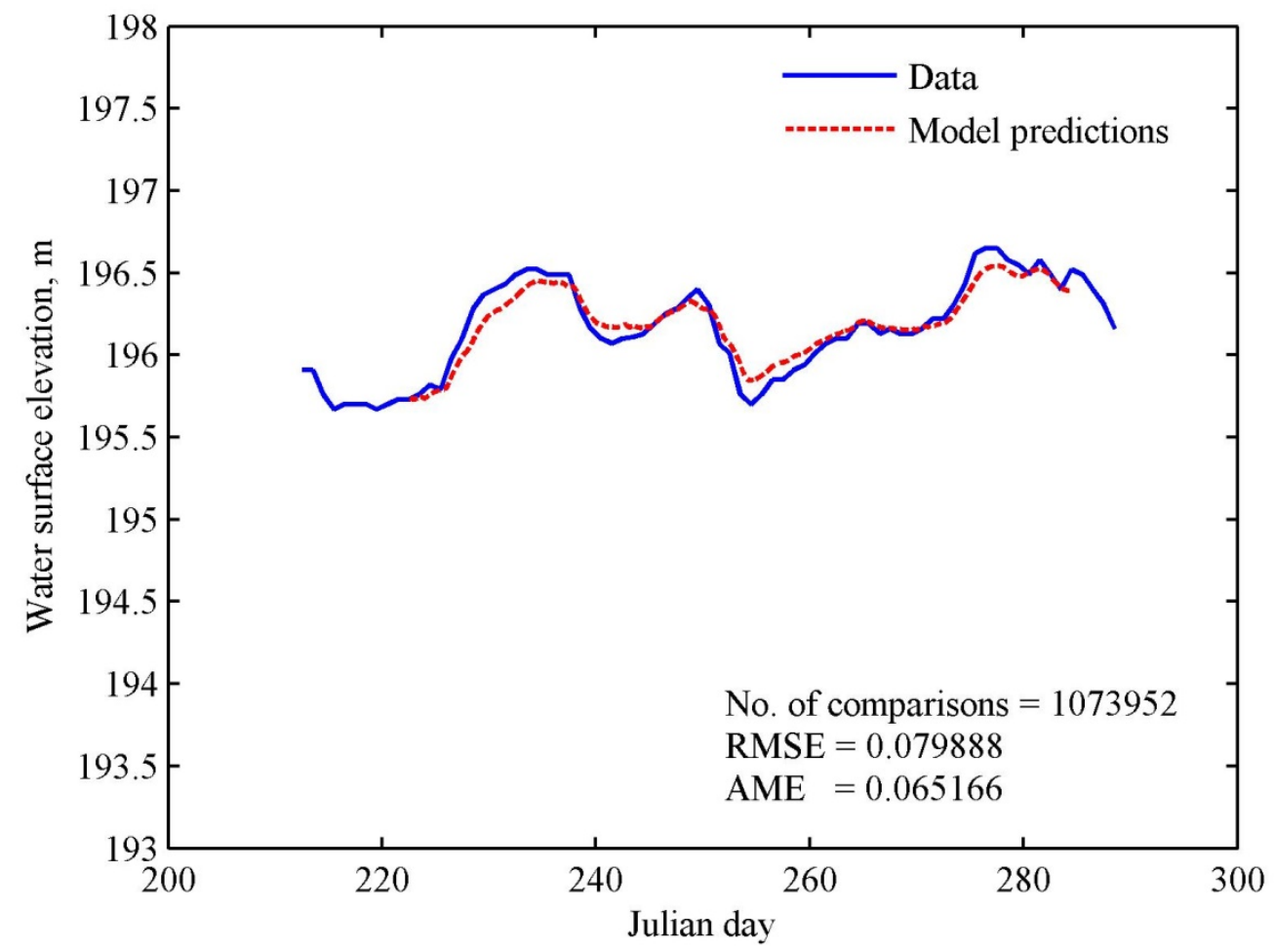

Figure 21 Model predictions of water surface elevation compared to field data 

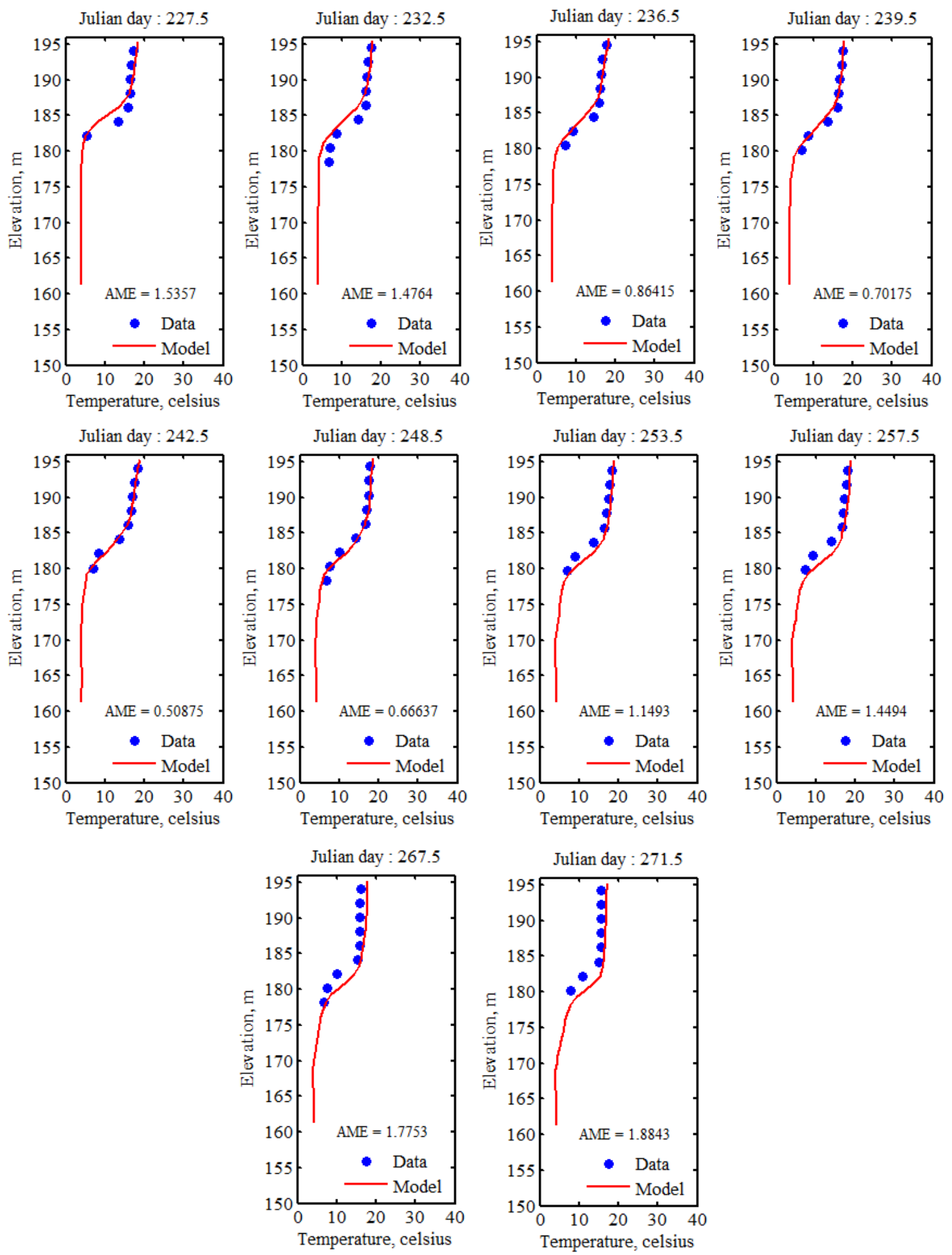

Figure 22 Model predictions of temperature compared to field data 